\title{
ANALYTIC TORSION AND R-TORSION FOR UNIMODULAR REPRESENTATIONS
}

\author{
WERNER MÜLLER
}

\section{CONTENTS}

0 . Introduction

1. Reidemeister torsion

2. Analytic torsion for unimodular representations

3. The equality of analytic torsion and R-torsion

4. Some examples

4.1. Locally symmetric manifolds

4.2. Chern-Simons gauge theory

References

\section{INTRODUCTION}

Let $M$ be a closed $C^{\infty}$-manifold of dimension $n$. Both $\mathrm{R}$-torsion and analytic torsion are smooth invariants of acyclic orthogonal (or unitary) representations $\rho$ of the fundamental group $\pi_{1}(M)$. The Reidemeister-Franz torsion (or R-torsion) $\tau_{M}(\rho)$ of $\rho$ is defined in terms of the combinatorial structure of $M$ given by its smooth triangulations. The analytic torsion $T_{M}(\rho)$ was introduced by Ray and Singer [RS] as an analytic counterpart of R-torsion. In order to define the analytic torsion one has to choose a Riemannian metric on $M$. Then $T_{M}(\rho)$ is a certain weighted alternating product of regularized determinants of the Laplacians on differential $q$-forms of $M$ with values in the flat bundle $E_{\rho}$ defined by $\rho$. It was conjectured by Ray and Singer [RS] that $T_{M}(\rho)=\tau_{M}(\rho)$ for all acyclic orthogonal (or unitary) representations $\rho$. This conjecture was proved independently by Cheeger [C] and the author [Mü].

The restriction to orthogonal (or unitary) representations is certainly a limitation of the applicability of this result if $\pi_{1}(M)$ is infinite because an infinite discrete group will have, in general, many nonorthogonal finite-dimensional representations. It is the purpose of the present paper to remove this limitation.

We call a representation $\rho: \pi_{1}(M) \rightarrow \mathrm{GL}(E)$ on a finite-dimensional real or complex vector space $E$ unimodular if $|\operatorname{det} \rho(\gamma)|=1$ for all $\gamma \in \pi_{1}(M)$. Then we define $\mathrm{R}$-torsion and analytic torsion for unimodular representations, and the main result is that for odd-dimensional manifolds $M$ the equality of the two torsions extends to all unimodular representations.

Received by the editors September 11, 1991 and, in revised form, September 17, 1992.

1991 Mathematics Subject Classification. Primary 58G11, 58G25. 
Now we shall explain this in more detail. Let $\rho: \pi_{1}(M) \rightarrow \mathrm{GL}(E)$ be an acyclic unimodular representation. Then the definition of $\mathrm{R}$-torsion also makes sense for $\rho$. Nothing has to be changed. The problem is how to define the analytic torsion. If $\rho$ is an orthogonal (or unitary) representation, then the flat bundle $E_{\rho}$ over $M$ defined by $\rho$ can be equipped with a natural metric which is compatible with the flat connection. Associated to the metrics on $E_{\rho}$ and $M$ is the Laplacian $\Delta_{q}$ acting on the space $\Lambda^{q}\left(M ; E_{\rho}\right)$ of $E_{\rho}$-valued differential $q$-forms on $M$. The zeta function of $\Delta_{q}$ is defined in the usual way by

$$
\zeta_{q}(s ; \rho)=\sum_{\lambda_{j}>0} \lambda_{j}^{-s}, \quad \operatorname{Re}(s)>n / 2,
$$

where the $\lambda_{j}$ run through the eigenvalues of $\Delta_{q}$. It is well known that this function has a meromorphic continuation to $\mathbf{C}$ which is holomorphic at $s=0$ [Se]. Then the definition of analytic torsion given by Ray and Singer is

$$
T_{M}(\rho)=\exp \left(\left.\frac{1}{2} \sum_{q=0}^{n}(-1)^{q} q \frac{d}{d s} \zeta_{q}(s ; \rho)\right|_{s=0}\right) .
$$

For acyclic representations, $T_{M}(\rho)$ is independent of the metric on $M$.

For an arbitrary finite-dimensional representation $\rho: \pi_{1}(M) \rightarrow \mathrm{GL}(E)$, there is no metric on $E_{\rho}$ which is compatible with the flat connection. In order to define the analytic torsion in this case we proceed as follows. We pick a metric $h$ on $E_{\rho}$. Using the metrics on $M$ and $E_{\rho}$, we define an inner product on $\Lambda^{*}\left(M ; E_{\rho}\right)$ which in turn gives rise to the Laplacian $\Delta_{h}$. Then we define the analytic torsion $T_{M}(\rho ; h)$ by formula $(0.1)$ where $\zeta_{q}(s ; \rho)$ is now the zeta function of the $q$-component of the new Laplacian $\Delta_{h}$.

We note that this approach was used by Schwarz [S] in his treatment of abelian Chern-Simons theory and that it is actually the origin for our definition of analytic torsion for nonorthogonal representations. We also note that a similar approach has recently been used by Bar-Natan and Witten [B-NW] to deal with the perturbative expansion of nonabelian Chern-Simons gauge theory with noncompact gauge group.

The first important result about analytic torsion in the present context is that, for an odd-dimensional manifold $M$ and an acyclic representation $\rho$, $T_{M}(\rho ; h)$ is independent of the choice of $h$ on $E_{\rho}$. Of course, it is also independent of the Riemannian metric on $M$ and we denote its common value, for any choice of $h$ and $g$, by $T_{M}(\rho)$. In general, the variation of $T_{M}(\rho ; h)$ with respect to $h$ and $g$ is given by an explicit formula. If $\operatorname{dim} M$ is even, the variational formula contains additional terms which are locally computable, that is, they are obtained by integrating densities which in any coordinate system are given by universal polynomials in the components $g_{i j}(x)$ and $h_{i j}(x)$ of the metrics and a finite number of their partial derivatives. There are examples showing that these terms may not vanish even if the representation is acyclic.

Using the inner product induced on the space of harmonic forms, we can also define the R-torsion $\tau_{M}(\rho ; h)$ for nonacyclic representations $\rho$.

On the first sight, the choice of an arbitrary metric on $E_{\rho}$ seems to be very artificial, but it is no more artificial than the arbitrary choice of the Riemannian 
metric on $M$. In general, there is no distinguished choice of a Riemannian metric on $M$. Only for special manifolds, like locally symmetric manifolds, do there exist distinguished metrics on $M$ which make analytic torsion for orthogonal representations accessible to computations. In this case, however, there exist also distinguished metrics on $E_{\rho}$ for many unimodular representations which makes analytic torsion for these representations also accessible to computations.

The main result of the present paper is

Theorem 1. Let $M$ be a closed smooth Riemannian manifold of odd dimension, and let $\rho: \pi_{1}(M) \rightarrow \mathrm{GL}(E)$ be a unimodular representation on a finitedimensional real (or complex) vector space. Let $E_{\rho}$ be the associated flat bundle. Then for any choice of a metric on $E_{\rho}$ we have $T_{M}(\rho ; h)=\tau_{M}(\rho ; h)$.

We remark that, in general, Theorem 1 does not hold in even dimensions.

To prove Theorem 1 we follow essentially Cheeger's proof [C]. First we show that $\log T_{M}(\rho ; h)-\log \tau_{M}(\rho ; h)$ is independent of the metrics $h$ on $E_{\rho}$ and $g$ on $M$. The main idea is then to keep track of

$$
e_{M}(\rho)=\log T_{M}(\rho ; h)-\log \tau_{M}(\rho ; h)
$$

if one does surgery on an embedded $k$-sphere in $M$ and finally to reduce everything to the case where one is able to show the equality explicitly. Cheeger's proof is well suited to work for nonorthogonal representations, because the decisive part of the proof consists of local analysis near a given handle of $M$ and the handles considered are such that $E_{\rho}$ restricted to any of them is trivial as a flat bundle. Since we are free to choose the metrics $h$ and $g$ according to our purpose, we choose $h$ and $g$ to be the standard product metrics near the given handle. Then all the local analysis done by Cheeger in [C] extends without change to the present situation.

Now we describe briefly the content of the paper. In $\S 1$ we review Reidemeister torsion for unimodular representations and we establish some of its properties. In $\S 2$ we introduce analytic torsion for finite-dimensional representations and we prove some of its properties. We also consider analytic torsion for manifolds with boundary and prove some results related to the variation of analytic torsion and R-torsion in this case. This is needed in $\S 3$ where we establish the equality of the two torsions for unimodular representations. We explain the main steps of the proof and refer to Cheeger's paper [C] for all details not discussed in this section. Finally, in $\S 4$ we consider two examples where nonorthogonal representations occur in nature and the analytic torsion as defined in $\S 2$ arises naturally in this context. The first example are compact locally symmetric manifolds. The results of Borel and Wallach [BW] can be used to obtain numerous examples of acyclic unimodular representations. The corresponding flat bundles can be equipped with canonical metrics which are locally homogeneous so that methods of harmonic analysis can be applied. The second example is Chern-Simons gauge theory. Witten [W1] has shown that, for a compact gauge group, the analytic torsion of flat connections occurs in the perturbative expansion of the path integral defined by the Chern-Simons action. 
This has been recently extended to noncompact gauge groups by Bar-Natan and Witten [B-NW], and we explain how it is related to Theorem 1.

\section{REIDEMEISTER TORSION}

In this section we study Reidemeister torsion for unimodular representations and collect some of its basic properties. Most of it is similar to the case of an orthogonal representation and we refer to [Mil] for details.

Given a real vector space $E$ of dimension $n$, we set

$$
\operatorname{det} E=\Lambda^{n}(E) \text {. }
$$

If $E=\{0\}$, then $\operatorname{det} E=\mathbf{R}$. Furthermore, if $L$ is a one-dimensional vector space, each nonzero element $l \in L$ determines a unique element $l^{-1} \in L^{*}$ defined by the equation $l^{-1}(l)=1$ and we shall use the notation $L^{-1}=L^{*}$.

A volume on $E$ will be a nonzero element $\omega \in \operatorname{det} E$. Any volume determines an isomorphism $\operatorname{det} E \cong \mathbf{R}$. Note also that a volume $\omega$ can be written as $e_{1} \wedge \cdots \wedge e_{n}$ for some basis $e_{1}, \ldots, e_{n}$ of $E$.

Let

$$
C_{\bullet}: 0 \rightarrow C_{n} \stackrel{\partial_{n}}{\longrightarrow} C_{n-1} \stackrel{\partial_{n-1}}{\longrightarrow} \cdots \stackrel{\partial_{1}}{\longrightarrow} C_{0} \rightarrow 0
$$

be a chain complex of finite-dimensional real vector spaces, and let

$$
H_{q}\left(C_{\bullet}\right)=\left(\operatorname{ker} \partial_{q}\right) / \operatorname{Im} \partial_{q+1}
$$

be the $q$ th homology group of $C_{\bullet}$. The determinant line of the complex $(1.1)$ is the one-dimensional vector space

$$
\operatorname{det}\left(C_{\bullet}\right)=\bigotimes_{q=0}^{n}\left(\operatorname{det} C_{q}\right)^{(-1)^{q}}
$$

We also set

$$
\operatorname{det} H_{*}\left(C_{\bullet}\right)=\bigotimes_{q=0}^{n}\left(\operatorname{det} H_{q}\left(C_{\bullet}\right)\right)^{(-1)^{q}} .
$$

Let $b_{q}=\operatorname{dim} \partial\left(C_{q}\right)$ and $h_{q}=\operatorname{dim} H_{q}\left(C_{\bullet}\right)$. For each $q, 1 \leq q \leq n$, we choose $\theta_{q} \in \Lambda^{b_{q}}\left(C_{q}\right)$ such that $\partial \theta_{q} \neq 0$. Furthermore, let $0 \neq \mu_{q} \in \operatorname{det} H_{q}\left(C_{\bullet}\right)$ and $\nu_{q} \in \Lambda^{h_{q}}\left(\operatorname{ker} \partial_{q}\right)$ be such that $\pi\left(\nu_{q}\right)=\mu_{q}$ where $\pi: \operatorname{ker} \partial_{q} \rightarrow H_{q}\left(C_{\bullet}\right)$ is the canonical projection. Let $i: \operatorname{ker} \partial_{q} \rightarrow \mathscr{C}_{q}$ be the natural embedding. Then $\partial \theta_{q+1} \wedge \theta_{q} \wedge i\left(\nu_{q}\right)$ is a nonzero element of $\operatorname{det} C_{q}$. Set $\mu=\bigotimes_{q=0}^{n}\left(\mu_{q}\right)^{(-1)^{q+1}}$. Then we define the torsion

$$
T\left(C_{\bullet}\right) \in\left(\operatorname{det} C_{\bullet}\right) \otimes\left(\operatorname{det} H_{*}\left(C_{\bullet}\right)\right)^{-1}
$$

of the complex (1.1) by

$$
\begin{aligned}
T\left(C_{\bullet}\right)= & \left(\partial \theta_{1} \wedge i\left(\nu_{0}\right)\right) \otimes\left(\partial \theta_{2} \wedge \theta_{1} \wedge i\left(\nu_{1}\right)\right)^{-1} \otimes \cdots \\
& \otimes\left(\partial \theta_{n} \wedge \theta_{n-1} \wedge i\left(\nu_{n-1}\right)\right)^{(-1)^{n-1}} \otimes\left(\theta_{n} \wedge i\left(\nu_{n}\right)\right)^{(-1)^{n}} \otimes \mu .
\end{aligned}
$$


It is easy to see that $T\left(C_{\bullet}\right)$ is independent of the particular choices of $\theta_{q}, \mu_{q}$, and $\nu_{q}$.

Now assume that we have chosen $0 \neq \omega \in \operatorname{det} C_{\bullet}$. Then $\omega$ defines an isomorphism $\operatorname{det} C_{\bullet} \cong \mathbf{R}$ and, therefore, also an isomorphism

$$
\operatorname{det} C_{\bullet} \otimes\left(\operatorname{det} H_{*}\left(C_{\bullet}\right)\right)^{-1} \cong\left(\operatorname{det} H_{*}\left(C_{\bullet}\right)\right)^{-1} \text {. }
$$

The image of $T\left(C_{\bullet}\right)$ with respect to this isomorphism will be denoted by

$$
T\left(C_{\bullet}, \omega\right) \in\left(\operatorname{det} H_{*}\left(C_{\bullet}\right)\right)^{-1} \text {. }
$$

Note that for a 2-term complex $C_{\bullet}: 0 \rightarrow C_{0} \stackrel{A}{\rightarrow} C_{1} \rightarrow 0$ with $C_{0}=C_{1}=\mathbf{R}^{N}$ and $\omega_{q} \in \operatorname{det} C_{q}$ being the canonical volumes , $T\left(C_{\bullet}, \omega\right)=\operatorname{det} A$.

Definition 1.4. The element $T\left(C_{\bullet}, \omega\right) \in\left(\operatorname{det} H_{*}\left(C_{\bullet}\right)\right)^{-1}$ defined above is called the Reidemeister torsion (or R-torsion) of $C_{\text {. }}$ with respect to the volume $\omega$.

If $C_{\bullet}$ is acyclic, i.e., $H_{*}\left(C_{\bullet}\right)=\{0\}$, then $\left(\operatorname{det} H_{*}\left(C_{\bullet}\right)\right)^{-1}=\mathbf{R}$ and $T\left(C_{\bullet}, \omega\right)$ is a real number. More generally, any choice of a volume $\mu \in \operatorname{det} H_{*}\left(C_{\bullet}\right)$ induces a natural isomorphism $\left(\operatorname{det} H_{*}\left(C_{\bullet}\right)\right)^{-1} \cong \mathbf{R}$ by sending $\lambda \mu^{-1}$ to $\lambda \in$ $\mathbf{R}$.Thus $T\left(C_{\bullet}, \omega\right)$ can be identified with a real number $T\left(C_{\bullet}, \omega, \mu\right) \in \mathbf{R}$ and we set

$$
\tau\left(C_{\bullet}, \omega, \mu\right)=\left|T\left(C_{\bullet}, \omega, \mu\right)\right|
$$

Next we discuss some of the properties satisfied by R-torsion.

Let $C_{\bullet}$ and $C_{\bullet}^{\prime}$ be two chain complexes with volumes $\omega \in \operatorname{det} C_{\bullet}, \omega^{\prime} \in$ $\operatorname{det} C_{\bullet}^{\prime}, \mu \in \operatorname{det} H_{*}\left(C_{\bullet}\right)$, and $\mu^{\prime} \in \operatorname{det} H^{*}\left(C_{\bullet}^{\prime}\right)$. Let $C_{\bullet} \otimes C_{\bullet}^{\prime}$ denote the tensor product complex with its standard differential, i.e.,

$$
\left(C_{\bullet} \otimes C_{\bullet}^{\prime}\right)_{q}=\bigoplus_{r+s=q}\left(C_{r} \otimes C_{s}^{\prime}\right)
$$

Then we have a natural isomorphism

$$
\operatorname{det}\left(C_{\bullet} \otimes C_{\bullet}^{\prime}\right) \cong\left(\operatorname{det} C_{\bullet}\right)^{\otimes \chi\left(C_{\bullet}^{\prime}\right)} \otimes\left(\operatorname{det} C_{\bullet}^{\prime}\right)^{\otimes \chi\left(C_{\bullet}\right)} .
$$

Let $\omega \otimes \omega^{\prime}$ denote the element of $\operatorname{det}\left(C_{\bullet} \otimes C_{\bullet}^{\prime}\right)$ which corresponds to $\omega^{\otimes \chi\left(C_{\bullet}^{\prime}\right)} \otimes$ $\left(\omega^{\prime}\right)^{\otimes x\left(C_{\bullet}\right)}$ under this isomorphism. The Kunneth formula induces another natural isomorphism

$$
\operatorname{det} H_{*}\left(C_{\bullet} \otimes C_{\bullet}^{\prime}\right) \cong \operatorname{det} H_{*}\left(C_{\bullet}\right)^{\otimes \chi\left(C_{\bullet}^{\prime}\right)} \otimes \operatorname{det} H_{*}\left(C_{\bullet}^{\prime}\right)^{\otimes \chi\left(C_{\bullet}\right)} .
$$

We let $\mu \otimes \mu^{\prime}$ denote the element of $\operatorname{det} H_{*}\left(C_{\bullet} \otimes C_{\bullet}^{\prime}\right)$ which corresponds to $\mu^{\otimes \chi\left(C_{\bullet}^{\prime}\right)} \otimes\left(\mu^{\prime}\right)^{\otimes \chi\left(C_{\bullet}\right)}$ under this isomorphism.

Proposition 1.6. The following equality holds:

$$
\tau\left(C_{\bullet} \otimes C_{\bullet}^{\prime}, \omega \otimes \omega^{\prime}, \mu \otimes \mu^{\prime}\right)=\tau\left(C_{\bullet}, \omega, \mu\right)^{\chi\left(C_{\bullet}^{\prime}\right)} \tau\left(C_{\bullet}^{\prime}, \omega^{\prime}, \mu^{\prime}\right)^{\chi\left(C_{\bullet}\right)}
$$

where $\chi\left(E_{\bullet}\right)$ denotes the Euler characteristic of the complex $E_{\bullet}$.

Proof. We introduce inner products on $C_{\bullet}, C_{\bullet}^{\prime}, H_{*}\left(C_{\bullet}\right)$, and $H_{*}\left(C_{\bullet}^{\prime}\right)$ inducing the corresponding volumes. These inner products define inner products on 
$C_{\bullet} \otimes C_{\bullet}^{\prime}$ and $H_{*}\left(C_{\bullet} \otimes C_{\bullet}^{\prime}\right)$ in the canonical way. It is easy to see that the induced volumes are $\omega \otimes \omega^{\prime} \in \operatorname{det}\left(C_{\bullet} \otimes C_{\bullet}^{\prime}\right)$ and $\mu \otimes \mu^{\prime} \in \operatorname{det} H_{*}\left(C_{\bullet} \otimes C_{\bullet}^{\prime}\right)$. Now we can proceed as in Proposition 1.16 in [C]. Q.E.D.

Let

$$
C_{\bullet}^{*}: 0 \leftarrow C_{n}^{*} \stackrel{\partial_{n}^{*}}{\longleftarrow} C_{n-1}^{*} \stackrel{\partial_{n-1}^{*}}{\longleftarrow} \ldots \stackrel{\partial_{1}^{*}}{\longleftarrow} C_{0}^{*} \leftarrow 0
$$

be the dual complex to (1.1). Note that there are natural isomorphisms

$$
\operatorname{det} C_{q}^{*} \cong\left(\operatorname{det} C_{q}\right)^{-1} \text { and } \operatorname{det} H_{q}\left(C_{\bullet}^{*}\right) \cong\left(\operatorname{det} H_{q}\left(C_{\bullet}\right)\right)^{-1} .
$$

Hence, we get a natural isomorphism

$$
\left(\operatorname{det} C_{\bullet} \otimes\left(\operatorname{det} H_{*}\left(C_{\bullet}\right)\right)^{-1}\right) \otimes\left(\operatorname{det} C_{\bullet}^{*} \otimes\left(\operatorname{det} H_{*}\left(C_{\bullet}^{*}\right)\right)^{-1}\right)^{(-1)^{n}} \cong \mathbf{R} .
$$

Proposition 1.8. With respect to the isomorphism (1.7), we have

$$
T\left(C_{\bullet}\right) \otimes T\left(C_{\bullet}^{*}\right)^{(-1)^{n}}= \pm 1 .
$$

The proof is straightforward and follows by an easy generalization of the argument used on p. 141 of [Mi2] to prove equality (3).

Let $\omega \in \operatorname{det} C_{\text {. }}$ and $\mu \in \operatorname{det} H_{*}\left(C_{\bullet}\right)$ be volumes. Denote by $\omega^{*} \in \operatorname{det} C_{\bullet}^{*} \cong$ $\left(\operatorname{det} C_{\bullet}\right)^{-1}$ and $\mu^{*} \in \operatorname{det} H_{*}\left(C_{\bullet}^{*}\right) \cong\left(\operatorname{det} H_{*}\left(C_{\bullet}\right)\right)^{*}$ the dual volumes determined by $\omega$ and $\mu$, respectively.

Corollary 1.9. We have the equality

$$
\tau\left(C_{\bullet}, \omega, \mu\right) \tau\left(C_{\bullet}^{*}, \omega^{*}, \mu^{*}\right)^{(-1)^{n}}=1 .
$$

The R-torsion arises in the following context. Let $K$ be a finite cell complex and $\widetilde{K}$ the universal covering space of $K$ with the fundamental group $\pi_{1}=$ $\pi_{1}(K)$ acting as deck transformations on $\widetilde{K}$. We think of $K$ as being embedded as a fundamental domain in $\widetilde{K}$, so that $\widetilde{K}$ is the union of the translates of $K$ under $\pi_{1}$. Let $C_{q}(\widetilde{K})$ be the real chain group generated by the $q$-cells of $\widetilde{K}$. Then $C_{q}(\widetilde{K})$ is a module over the real group algebra $\mathbf{R}\left(\pi_{1}\right)$. The $q$-cells of $K$ form a preferred base for $C_{q}(\widetilde{K})$ as $\mathbf{R}\left(\pi_{1}\right)$-module. Let $\rho: \pi_{1} \rightarrow \mathrm{GL}(E)$ be a representation of $\pi_{1}$ on a real vector space $E$ of dimension $N$. It defines a flat bundle $E_{\rho}$ over $K$ and we define the chain group $C_{q}(K ; E)$ of chains with values in the local system $E_{\rho}$ by $C_{q}(K ; E)=C_{q}(\widetilde{K}) \otimes_{\mathbf{R}\left(\pi_{1}\right)} E$. The boundary operator $\widetilde{\partial}_{q}: C_{q}(\widetilde{K}) \rightarrow C_{q-1}(\widetilde{K})$ induces $\partial_{q}: C_{q}(K ; E) \rightarrow C_{q-1}(K ; E)$ and we get a real chain complex

$$
C_{\bullet}(K ; E): 0 \rightarrow C_{n}(K ; E) \stackrel{\partial_{n}}{\longrightarrow} C_{n-1}(K ; E) \stackrel{\partial_{n-1}}{\longrightarrow} \cdots \stackrel{\partial_{1}}{\longrightarrow} C_{0}(K ; E) \rightarrow 0 ;
$$

its homology will be denoted by $H_{*}(K ; E)$. Similarly, we have the cochain complex with coefficients in $E_{\rho}$ :

$$
C^{\bullet}(K ; E): 0 \rightarrow C^{0}(K ; E) \stackrel{\delta_{1}}{\longrightarrow} C^{1}(K ; E) \stackrel{\delta_{2}}{\longrightarrow} \cdots \stackrel{\delta_{n}}{\longrightarrow} C^{n}(K ; E) \rightarrow 0
$$


where $C^{q}(K ; E)=\operatorname{Hom}_{\mathbf{R}\left(\pi_{1}\right)}\left(C_{q}(\widetilde{K}), E\right)$ and $\delta=\partial^{*}$. Note that

$$
C^{q}(K ; E) \cong C^{q}(\widetilde{K}) \underset{\mathbf{R}\left(\pi_{1}\right)}{\otimes} E .
$$

We shall denote the cohomology of this complex by $H^{*}(K ; E)$.

Now assume that a volume $\theta \in \operatorname{det} E$ is given. Let $e_{j}^{q}, j=1, \ldots, r_{q}$, be the oriented $q$-cells of $K$ considered as a preferred base of the $\mathbf{R}\left(\pi_{1}\right)$-module $C_{q}(\widetilde{K})$, and let $x_{1}, \ldots, x_{N}$ be a base of $E$ such that $\theta= \pm x_{1} \wedge \cdots \wedge x_{N}$. Then $\left(e_{j}^{q} \otimes x_{k}\right)$ is a preferred base of $C_{q}(K ; E)$ and it defines a volume $\omega_{q} \in$ $\operatorname{det} C_{q}(K ; E)$.

At this point, the volumes depend on several choices, namely,

(a) the choice of the embedding of $K$ in the covering space $\widetilde{K}$;

(b) the orientation and ordering of the cells of $K$,

(c) the choice of the base $x_{1}, \ldots, x_{N}$ of $E$.

To deal with (a), we make the following

Definition 1.10. A representation $\rho: \pi_{1}(K) \rightarrow \mathrm{GL}(E)$ is called unimodular if $|\operatorname{det} \rho(\gamma)|=1$ for all $\gamma \in \pi_{1}(K)$.

If we assume that $\rho$ is unimodular, then a different choice of the embedding of $K$ into $\widetilde{K}$ corresponds to a change of base in $C_{q}(K ; E)$ by a matrix whose determinant is of absolute value one; therefore, the volume $\omega_{q}$ changes at most by sign. Similarly, (b) and (c) cause only a change of sign. Set $\omega=$ $\bigotimes_{q=0}^{n}\left(\omega_{q}\right)^{(-1)^{q}}$.

Remark. The volume $\omega$ depends, of course, on the choice of the volume $\theta$ on $E$. We indicate this by $\omega(\theta)$. If we replace $\theta$ by $\lambda \theta, \lambda \in \mathbf{R}$, then

$$
\omega(\lambda \theta)=\prod_{q=0}^{n} \lambda^{(-1)^{q} r_{q}} \omega(\theta)=\lambda^{\chi(K)} \omega(\theta)
$$

where $\chi(K)$ is the Euler characteristic of $K$. Thus, if $K$ is a triangulation of a closed oriented manifold of odd dimension, then $\omega$ is independent of the choice of a volume on $E$. If $\chi(K) \neq 0$, we fix a volume on $E$ and set $\omega=\omega(\theta)$.

Then we define the R-torsion $\tau_{K}(\rho)$ by

$$
\tau_{K}(\rho)=\left[\tau\left(C_{\bullet}(K ; E), \omega\right)\right] \in\left(\operatorname{det} H_{*}(K ; E)\right)^{-1} /\{ \pm 1\}
$$

and this definition does not depend on the choices that we made to define the volume element $\omega$ for $C_{\bullet}(K ; E)$. Let $\mu \in \operatorname{det} H_{*}(K ; E)$ be a volume. Then we have a natural isomorphism

$$
\left(\operatorname{det} H_{*}(K ; E)\right)^{-1} /\{ \pm 1\} \cong \mathbf{R}^{+},
$$

and $\tau_{K}(\rho)$ can be identified with a positive real number which we denote by $\tau_{K}(\rho ; \mu)$. An important fact is that $\tau_{K}(\rho ; \mu)$ is invariant under subdivision. The proof is similar to the corresponding proof for orthogonal representations in [Mil]. Thus $\tau_{K}(\rho)$ is a combinatorial invariant. 
Let $M$ be a compact smooth manifold, and let $\rho: \pi_{1}(M) \rightarrow \mathrm{GL}(E)$ be a unimodular representation. The manifold $M$ has a distinguished class of triangulations, the so-called smooth triangulations. Any two of these have a common subdivision (cf. [Mu]). Thus, for any choice of a volume $\mu \in \operatorname{det} H_{*}(M)$, we get an invariant $\tau_{M}(\rho ; \mu) \in \mathbf{R}^{+}$.

Definition 1.11. The positive real number $\tau_{M}(\rho ; \mu)$ is called the Reidemeister torsion (or $R$-torsion) of the manifold $M$ with respect to $\rho$ and $\mu$.

Recall that a representation $\rho: \pi_{1}(M) \rightarrow \mathrm{GL}(E)$ is called acyclic if $H^{*}\left(M ; E_{\rho}\right)=0$.

If $\rho$ is acyclic, the R-torsion $\tau_{M}(\rho)$ is a positive real number which is an invariant of the manifold $M$ and the representation $\rho$.

As above, the R-torsion $\tau_{M}(\rho ; \mu)$ has several important properties. Here we recall two of them which we need in $\S 3$ to prove the equality of analytic torsion and R-torsion.

Let $M$ be a closed orientable manifold of dimension $n$, and let $\rho: \pi_{1}(M) \rightarrow$ $\mathrm{GL}(E)$ be a unimodular representation with associated flat bundle $E_{\rho}$. Let $\rho^{*}$ be the contragredient representation. Then $\rho^{*}$ is also unimodular and the associated flat bundle is the dual vector bundle $E_{\rho}^{*}$ of $E_{\rho}$. For each $q$, the Poincaré duality isomorphism induces an isomorphism

$$
\lambda_{q}: \operatorname{det} H_{q}\left(M ; E^{*}\right) \stackrel{\sim}{\longrightarrow}\left(\operatorname{det} H_{n-q}(M ; E)\right)^{-1} .
$$

The following result is a slight extension of Milnor's duality theorem [Mi2].

Proposition 1.12. Assume that, for each $q$, we have chosen volumes $\mu_{q} \in$ $\operatorname{det} H_{q}(M ; E)$ and $\mu_{q}^{*} \in \operatorname{det} H_{q}\left(M ; E^{*}\right)$ satisfying $\lambda_{q}\left(\mu_{q}^{*}\right)=\left(\mu_{n-q}\right)^{-1}$. Then we have

$$
\tau_{M}(\rho ; \mu) \tau_{M}\left(\rho^{*} ; \mu^{*}\right)^{(-1)^{n}}=1
$$

Let $M_{1}, M_{2}$ be two compact smooth manifolds, and let $\rho_{i}$ : $\pi_{1}\left(M_{i}\right) \rightarrow \mathrm{GL}\left(E_{i}\right), i=1,2$, be unimodular representations with associated flat bundles $E_{\rho_{1}}, E_{\rho_{2}}$. Note that $\pi_{1}\left(M_{1} \times M_{2}\right)=\pi_{1}\left(M_{1}\right) \times \pi_{1}\left(M_{2}\right)$ and the representation $\rho_{1} \otimes \rho_{2}: \pi_{1}\left(M_{1} \times M_{2}\right) \rightarrow \mathrm{GL}\left(E_{1} \otimes E_{2}\right)$ is unimodular. Denote by $p_{i}: M_{1} \times M_{2} \rightarrow M_{i}, i=1,2$, the canonical projection. Then the flat bundle over $M_{1} \times M_{2}$ defined by $\rho_{1} \otimes \rho_{2}$ equals $p_{1}^{*} E_{\rho_{1}} \otimes p_{2}^{*} E_{\rho_{2}}$. Let $\mu_{i} \in \operatorname{det} H_{*}\left(M_{i} ; E_{i}\right), i=1,2$, be volumes. By the Kunneth formula we obtain an isomorphism

$$
\begin{aligned}
\operatorname{det} H_{*}\left(M_{1} \times M_{2} ; E_{1} \otimes E_{2}\right) & \\
= & \operatorname{det} H_{*}\left(M_{1} ; E_{1}\right)^{\chi\left(M_{2} ; E_{2}\right)} \otimes \operatorname{det} H_{*}\left(M_{2} ; E_{2}\right)^{\chi\left(M_{1} ; E_{1}\right)} .
\end{aligned}
$$

Let $\mu_{1} \otimes \mu_{2}$ be the element of $\operatorname{det} H_{*}\left(M_{1} \times M_{2} ; E_{1} \otimes E_{2}\right)$ which corresponds to $\mu_{1}^{\chi\left(M_{2} ; E_{2}\right)} \otimes \mu_{2}^{\chi\left(M_{1} ; E_{1}\right)}$ under this isomorphism. As a consequence of Proposition 1.6 we get

Proposition 1.13. With the notation above, we have the equality

$$
\tau_{M_{1} \times M_{2}}\left(\rho_{1} \otimes \rho_{2} ; \mu_{1} \otimes \mu_{2}\right)=\tau_{M_{1}}\left(\rho_{1} ; \mu_{1}\right)^{\chi\left(M_{2} ; E_{2}\right)} \cdot \tau_{M_{2}}\left(\rho_{2} ; \mu_{2}\right)^{\chi\left(M_{1} ; E_{1}\right)} .
$$


The volumes $\mu$ that we are considering arise in the following way. We choose metrics on $M$ and on the flat bundle $E_{\rho}$ defined by $\rho$. If $\partial M \neq \varnothing$ we also impose boundary conditions for the Laplacian on forms. Then the space of harmonic forms has an inner product and, via the De Rham isomorphism combined with Poincaré duality, we get inner products in $H_{*}(M ; E)$ or $H_{*}(M, \partial M ; E)$, depending on the boundary conditions. These inner products define volumes. In particular, for this choice of volumes the condition of Proposition 1.12 is satisfied.

Remark. We may also work with complex coefficients and define the R-torsion in this case. As above, a representation $\rho: \pi_{1}(M) \rightarrow \mathrm{GL}(E)$ on a complex finite-dimensional vector space $E$ is called unimodular if $|\operatorname{det} \rho(\gamma)|=1$ for all $\gamma \in \pi_{1}(M)$. For a unimodular representation $\rho$ the $\mathrm{R}$-torsion $\tau_{M}(\rho ; \mu)$ is defined in the same way as in the real case.

Let $i: \mathrm{GL}(N, \mathbf{C}) \rightarrow \mathrm{GL}(2 N, \mathbf{R})$ be the standard embedding which sends $g=A+\sqrt{-1} B, A, B \in \operatorname{Mat}(N, \mathbf{R})$ to $\left(\begin{array}{cc}A-B \\ B & A\end{array}\right) \in \mathrm{GL}(2 N, \mathbf{R})$. If $|\operatorname{det}(g)|=1$ then $|\operatorname{det}(i(g))|=1$. Hence, if $\rho: \pi_{1}(M) \rightarrow \mathrm{GL}(N, \mathbf{C})$ is unimodular, then $i \circ \rho: \pi_{1}(M) \rightarrow \mathrm{GL}(2 N, \mathbf{R})$ is also unimodular and we are back to our previous framework.

\section{ANALYTIC TORSION FOR UNIMODULAR REPRESENTATIONS}

In this section we define the analytic torsion and prove some of its properties. Let $(M, g)$ be a compact Riemannian manifold of dimension $n$. For simplicity we assume that $M$ is orientable. Let $\rho: \pi_{1}(M) \rightarrow \mathrm{GL}(E)$ be a representation of the fundamental group of $M$ on a real vector space of dimension $N$. The representation $\rho$ defines a flat vector bundle $E_{\rho}$ over $M$. We choose a metric $h$ on $E_{\rho}$. It induces a R-linear isomorphism \# : $E_{\rho} \stackrel{\sim}{\longrightarrow} E_{\rho}^{*}$ where $E_{\rho}^{*}$ is the dual vector bundle. Let $\Lambda^{p}(E)$ be the space of $C^{\infty} p$-forms on $M$ with values in $E_{\rho}$, i.e., the space of smooth sections of the vector bundle $\Lambda^{p}\left(T^{*} M\right) \otimes E_{\rho}$. Then \# extends to an isomorphism

$$
\#: \Lambda^{p}(E) \stackrel{\sim}{\longrightarrow} \Lambda^{p}\left(E^{*}\right)
$$

for each $p$. Furthermore, the Riemannian metric on $M$ defines a linear mapping

$$
*: \Lambda^{p}(E) \longrightarrow \Lambda^{n-p}(E)
$$

for each $p$ (cf. [MM, §2]) which satisfies $* *=(-1)^{p(n-p)}$ on $\Lambda^{p}(E)$, and it is easy to see that $*$ and \# commute. The usual exterior product of differential forms combined with the evaluation map $\operatorname{tr}: E_{\rho} \otimes E_{\rho}^{*} \rightarrow \mathbf{R}$ induces the following exterior product for vector-valued forms

$$
\Lambda: \Lambda^{p}(E) \otimes \Lambda^{q}\left(E^{*}\right) \longrightarrow \Lambda^{p+q}(M)
$$

where $\Lambda^{*}(M)$ is the space of smooth differential forms on $M$ (cf. [MM, §2]). Then an inner product on $\Lambda^{p}(E)$ is defined by

$$
\left\langle\omega, \omega^{\prime}\right\rangle=\int_{M} \omega \wedge * \circ \# \omega^{\prime} .
$$


Let $L^{2} \Lambda^{p}(E)$ denote the completion of $\Lambda^{p}(E)$ with respect to the norm defined by this inner product. Since $E_{\rho}$ is flat, we have the De Rham complex

$$
\Lambda^{0}(E) \stackrel{d_{0}}{\longrightarrow} \Lambda^{1}(E) \stackrel{d_{1}}{\longrightarrow} \cdots \stackrel{d_{n-1}}{\longrightarrow} \Lambda^{n}(E) .
$$

The formal adjoint of $d_{p}$ on $\Lambda^{p}(E)$ is given by

$$
\delta_{p}=(-1)^{n p+n+1} * \text { o\# }^{-1} d_{p} \# \circ * \text {. }
$$

Remark. We may, of course, combine $*$ and \# into a single operator $\tilde{*}$. The advantage of this notation is that the dependence on the Riemannian metric on $M$ and on the Euclidean metric on $E_{\rho}$ is separated by $*$ and \#.

Now we define the Laplacian on $p$-forms as usual by

$$
\Delta_{p}=\delta_{p} d_{p}+d_{p-1} \delta_{p-1}
$$

Note that $\Delta_{p}$ does not depend on the choice of an orientation on $M$, and therefore we can define $\Delta_{p}$ also if $M$ is not orientable. One has to use the formalism of densities (see [C, $\S 3]$ for details).

Now assume that $\partial M=\varnothing$. Then the Laplacian is a symmetric, positive semidefinite, elliptic operator with pure point spectrum

$$
0 \leq \lambda_{0} \leq \lambda_{1} \leq \cdots \rightarrow \infty \text {. }
$$

Let $\mathscr{H}^{p}(E)$ denote the kernel of $\Delta_{p}$. This is the space of $E_{\rho}$-valued harmonic $p$-forms on $M$, and the De Rham map induces an isomorphism

$$
\mathscr{H}^{p}(E) \stackrel{\sim}{\longrightarrow} H^{p}(M ; E)
$$

where $H^{p}(M ; E)$ is the cohomology of $M$ with coefficients in the flat bundle $E_{\rho}$. Let $P_{q}$ denote the orthogonal projection of $L^{2} \Lambda^{q}(E)$ onto the subspace $\mathscr{H}^{q}(E)$. The zeta function associated to the Laplacian $\Delta_{q}$ on $\Lambda^{q}(E)$ is defined by

$$
\zeta_{q}(s ; \rho)=\frac{1}{\Gamma(s)} \int_{0}^{\infty} t^{s-1} \operatorname{Tr}\left(e^{-t \Delta_{q}}-P_{q}\right) d t=\sum_{\lambda_{j}>0} \lambda_{j}^{-s}, \quad \operatorname{Re}(s)>n / 2 .
$$

It is proved in [Se] that $\zeta_{q}(s ; \rho)$ extends to a meromorphic function of $s \in \mathbf{C}$ which is holomorphic at $s=0$. As in the case of an orthogonal representation, we define the analytic torsion $T_{M}(\rho ; h)$ by

$$
T_{M}(\rho ; h)=\exp \left(\left.\frac{1}{2} \sum_{q=0}^{n}(-1)^{q} q \frac{d}{d s} \zeta_{q}(s ; \rho)\right|_{s=0}\right) .
$$

Note that $T_{M}(\rho ; h)$ also depends on the Riemannian metric $g$, but we do not indicate this dependence explicitly.

First we establish a formula for the variation of the analytic torsion with respect to the Riemannian and Euclidean structure. To obtain such a formula we may proceed essentially the same way as in [RS, Theorems 2.1 and 7.3]. Consider one-parameter families $h_{u}, g_{v}$ of metrics on $E_{\rho}$ and $M$, respectively. Let $\Delta_{q}(u, v)$ be the Laplacian with respect to $\left(h_{u}, g_{v}\right)$. Set

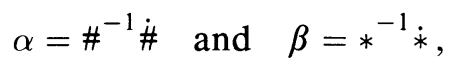


where

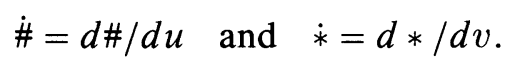

We regard $\alpha$ and $\beta$ as differential operators of order zero. Then it follows from Lemma 1.7.7 in [G] that, for $t \rightarrow 0$, there exist asymptotic expansions of the form

$$
\begin{aligned}
& \operatorname{Tr}\left(\alpha e^{-t \Delta_{q}(u, v)}\right) \sim \sum_{k=0}^{\infty} c_{q, k}(u, v) t^{-n / 2+k}, \\
& \operatorname{Tr}\left(\beta e^{-t \Delta_{q}(u, v)}\right) \sim \sum_{k=0}^{\infty} d_{q, k}(u, v) t^{-n / 2+k} .
\end{aligned}
$$

Theorem 2.6. Let $M$ be a closed oriented manifold of dimension $n$, and let $\rho$ be a representation of $\pi_{1}(M)$ on $E$. Let $h_{u}$ and $g_{v}$ be one-parameter families of metrics on $E_{\rho}$ and $M$, respectively. Let $P_{q}(u, v)$ denote the harmonic projection with respect to $\left(h_{u}, g_{v}\right)$. Furthermore, let $c_{q}(u, v)$ and $d_{q}(u, v)$ denote the constant terms of the asymptotic expansions (2.4) and (2.5), respectively. Then

$$
\begin{aligned}
& \frac{\partial}{\partial u} \log T_{M}(\rho ; u, v)=\frac{1}{2} \sum_{q=0}^{n}(-1)^{q} \operatorname{Tr}\left(\alpha_{u} P_{q}(u, v)\right)-\frac{1}{2} \sum_{q=0}^{n}(-1)^{q} c_{q}(u, v), \\
& \frac{\partial}{\partial v} \log T_{M}(\rho ; u, v)=\frac{1}{2} \sum_{q=0}^{n}(-1)^{q} \operatorname{Tr}\left(\beta_{v} P_{q}(u, v)\right)-\frac{1}{2} \sum_{q=0}^{n}(-1)^{q} d_{q}(u, v) .
\end{aligned}
$$

If $n$ is odd, the local terms $c_{q}$ and $d_{q}$ vanish and, by (2.1), we get

Corollary 2.7. Assume that $\operatorname{dim} M$ is odd and $\rho: \pi_{1}(M) \rightarrow \mathrm{GL}(E)$ is acyclic. Then $T_{M}(\rho ; h)$ has the same value for any choice of a Riemannian metric on $M$ and $a$ metric $h$ on $E_{\rho}$.

Thus, for an odd-dimensional manifold $M$ and an acyclic representation $\rho$, $T_{M}(\rho ; h)$ is an invariant $T_{M}(\rho)$ of the manifold $M$ and of the representation $\rho$. This justifies the following

Definition 2.8. Let $M$ be an odd-dimensional closed manifold and $\rho$ an acyclic representation of $\pi_{1}(M)$. Then the analytic torsion $T_{M}(\rho)$ of $\rho$ is the common value of $T_{M}(\rho ; h)$ for any choice of a metric $h$ on $E_{\rho}$.

Thus for closed manifolds of odd dimension our definition of analytic torsion works well. The situation is different if the dimension of $M$ is even. For orthogonal (or unitary) representations the individual local terms do not vanish in general, but their alternating sum does. This can be seen as follows. Assume that $E$ is equipped with an inner product and $\rho: \pi_{1}(M) \rightarrow \mathrm{GL}(E)$ preserves the inner product, that is, $\rho$ is orthogonal. Then $*$ commutes with $\Delta$. Hence

$$
e^{-t \Delta_{n-q} *^{-1}}=*^{-1} e^{-t \Delta_{q}} \text {. }
$$

Multiplying by $*$ and taking the trace gives

$$
\operatorname{Tr}\left(*^{-1} \dot{*} e^{-t \Delta_{n-q}}\right)=\operatorname{Tr}\left(e^{-t \Delta_{n-q}} *^{-1} \dot{*}\right)=\operatorname{Tr}\left(*^{-1} e^{-t \Delta_{q}} \dot{*}\right)=\operatorname{Tr}\left(\dot{*} *^{-1} e^{-t \Delta_{q}}\right) .
$$


Now observe that $\dot{*} *^{-1}=-*^{-1} \dot{*}$. Then

$$
\operatorname{Tr}\left(*^{-1} \dot{*} e^{-t \Delta_{n-q}}\right)=-\operatorname{Tr}\left(*^{-1} \dot{*} e^{-t \Delta_{q}}\right),
$$

which implies $d_{q, n / 2}=-d_{n-q, n / 2}$. Therefore, $\sum_{q=0}^{n}(-1)^{q} d_{q, n / 2}=0$.

This is false, however, if the metric on $E_{\rho}$ is not compatible with the flat connection. As an example consider a closed surface $\Sigma$ of genus $g \geq 2$ and choose on $\Sigma$ the metric of constant curvature -1 . Let $E_{\rho}$ be any flat orthogonal bundle over $\Sigma$. We denote by $\langle\cdot, \cdot\rangle$ the canonical metric on $E_{\rho}$ which is compatible with the flat connection. Let $f \in C^{\infty}(\Sigma)$ and $u \in \mathbf{R}$. Then we define a new metric $h_{u}$ by

$$
h_{u}(v, w)=e^{u f(z)}\langle v, w\rangle_{z} \text { for } v, w \in E_{\rho, z}, z \in \Sigma .
$$

If we use the canonical metric to identify $E_{\rho}$ and $E_{\rho}^{*}$, then $\#_{u}: E_{\rho} \rightarrow E_{\rho}$ is given by

$$
\#_{u} v=e^{u f(z)} v, \quad v \in E_{\rho, z}, z \in \Sigma .
$$

Hence $\alpha_{u}=\#^{-1} \#=f \cdot \mathrm{Id}$. Let $K_{q, u}\left(z, z^{\prime}, t\right)$ be the heat kernel on $E_{\rho}$-valued $q$-forms of $\Sigma$ where $E_{\rho}$ is equipped with $h_{u}$. Then

$$
\operatorname{tr}\left(\alpha_{u}(z) K_{q, u}(z, z, t)\right)=f(z) \operatorname{tr}\left(K_{q, u}(z, z, t)\right) .
$$

Let $m$ be the rank of $E_{\rho}$ and $K_{q}\left(z, z^{\prime}, t\right)$ the heat kernel for the Laplacian on $q$-forms. Then, for $u=0, \operatorname{tr} K_{q, 0}(z, z, t)=m \operatorname{tr} K_{q}(z, z, t)$. Furthermore, the first three coefficients of the asymptotic expansion of $\operatorname{tr} K_{q}(z, z, t)$ are well known (cf. [G, p. 330]). Since, by assumption, the curvature of $\Sigma$ is $\equiv-1$, it follows that

$$
c_{0}(0)-c_{1}(0)+c_{2}(0)=-\frac{m}{2 \pi} \int_{\Sigma} f(z) d z .
$$

This shows that in the even-dimensional case the local contribution to the variational formula for $T_{M}(\rho ; h)$ is nonzero in general. (See [B-Z] for further results about this problem.)

The analytic torsion for orthogonal representations satisfies a number of functorial properties (cf. [RS, §2]) which reflect known properties of the R-torsion. They continue to hold, with some modifications, for arbitrary finite-dimensional representations.

Let $E_{\rho}$ be a flat bundle over $M$ defined by the representation $\rho: \pi_{1}(M) \rightarrow$ $\mathrm{GL}(E)$. We denote by $\rho^{*}$ the contragredient representation. The associated flat bundle is the dual bundle $E_{\rho}^{*}$ of $E_{\rho}$. Any metric $h$ on $E_{\rho}$ induces a metric on $E_{\rho}^{*}$ which we denote by $h^{*}$.

Proposition 2.9. Suppose that $M$ is a closed orientable manifold of dimension $n$. Let $\rho$ be a finite-dimensional real representation of $\pi_{1}(M)$ and $E_{\rho}$ the associated flat vector bundle. For any choice of a metric $h$ on $E_{\rho}$, we have

$$
T_{M}(\rho ; h) T_{M}\left(\rho^{*} ; h^{*}\right)^{(-1)^{n}}=1 .
$$


Proof. We may essentially proceed as in the proof of Theorem 2.3 in [RS]. Let $\Delta_{\rho}\left(\right.$ resp. $\left.\Delta_{\rho^{*}}\right)$ denote the Laplacian on $E_{\rho}$-valued (resp. $E_{\rho^{*}}^{*}$-valued) differential forms of $M$. The zeta functions $\zeta_{q}(s ; \rho)$ and $\zeta_{q}\left(s ; \rho^{*}\right)$ of the operators $\Delta_{\rho, q}$ and $\Delta_{\rho^{*}, q}$, respectively, are defined as above. By (2.2), it suffices to show that

$$
\sum_{q=0}^{n}(-1)^{q} q\left(\zeta_{q}(s ; \rho)+(-1)^{n} \zeta_{q}\left(s ; \rho^{*}\right)\right) \equiv 0 .
$$

Let $\lambda>0$ be an eigenvalue of $\Delta_{\rho, q}$, and let $\mathscr{E}_{q}(\lambda ; \rho) \subset \Lambda^{q}(E)$ be the corresponding eigenspace. We introduce the following subspaces $\mathscr{E}_{q}^{\prime}(\lambda ; \rho)=\left\{\phi \in \mathscr{E}_{q}(\lambda ; \rho) \mid d \phi=0\right\} \quad$ and $\quad \mathscr{E}_{q}^{\prime \prime}(\lambda ; \rho)=\left\{\phi \in \mathscr{E}_{q}(\lambda ; \rho) \mid \delta \phi=0\right\}$. As in [RS, pp. 154-155], it follows that

$$
\mathscr{E}_{q}(\lambda ; \rho)=\mathscr{E}_{q}^{\prime}(\lambda ; \rho) \oplus \mathscr{E}_{q}^{\prime \prime}(\lambda ; \rho)
$$

and $\lambda^{-1 / 2} d$ defines an isometry of $\mathscr{E}_{q}^{\prime \prime}(\lambda ; \rho)$ onto $\mathscr{E}_{q+1}^{\prime}(\lambda ; \rho)$, with inverse $\lambda^{-1 / 2} \delta$. Let $m_{q}^{\prime}(\lambda ; \rho)$ and $m_{q}^{\prime \prime}(\lambda ; \rho)$ be the dimension of the spaces $\mathscr{E}_{q}^{\prime}(\lambda ; \rho)$ and $\mathscr{E}_{q}^{\prime \prime}(\lambda ; \rho)$, respectively. Then the multiplicity of $\lambda$ is

$$
m_{q}(\lambda ; \rho)=m_{q}^{\prime}(\lambda ; \rho)+m_{q}^{\prime \prime}(\lambda ; \rho)=m_{q}^{\prime}(\lambda ; \rho)+m_{q+1}^{\prime}(\lambda ; \rho)
$$

and we obtain

$$
\sum_{q=0}^{n}(-1)^{q} q \zeta_{q}(s ; \rho)=\sum_{q=1}^{n}(-1)^{q} \sum_{\lambda>0} m_{q}^{\prime}(\lambda ; \rho) \lambda^{-s}=-\sum_{q=0}^{n-1}(-1)^{q} \sum_{\lambda>0} m_{q}^{\prime \prime}(\lambda ; \rho) \lambda^{-s}
$$

and similarly for $\rho^{*}$. Let $d, \delta$ denote the differential and the codifferential with respect to $E_{\rho}$ and $d^{\prime}, \delta^{\prime}$ the corresponding operators with respect to $E_{\rho^{*}}=E_{\rho}^{*}$. Then $* \circ \#: \Lambda^{q}(E) \rightarrow \Lambda^{n-q}\left(E^{*}\right)$ satisfies $* \circ \# d \delta=\delta^{\prime} d^{\prime} *$ o\#. Hence, $* \circ \#$ defines an isometry of $\mathscr{E}_{q}^{\prime}(\lambda ; \rho)$ onto $\mathscr{E}_{n-q}^{\prime \prime}\left(\lambda ; \rho^{*}\right)$ showing that $m_{q}^{\prime}(\lambda ; \rho)=m_{n-q}^{\prime \prime}\left(\lambda ; \rho^{*}\right)$. Combined with (2.11), we obtain (2.10). Q.E.D.

Since $\left(H_{*}(M ; E)\right)^{*} \cong H_{*}\left(M ; E^{*}\right)$, the representation $\rho$ is acyclic iff the contragredient representation $\rho^{*}: \pi_{1}(M) \rightarrow \mathrm{GL}\left(E^{*}\right)$ is acyclic and we get

Corollary 2.12. Let $M$ be a closed orientable manifold of even dimension, and let $\rho: \pi_{1}(M) \rightarrow \mathrm{GL}(E)$ be an acylic representation. Then

$$
T_{M}(\rho) T_{M}\left(\rho^{*}\right)=1 .
$$

Assume that $E$ is equipped with an inner product so that $E \cong E^{*}$. Then $\rho$ is orthogonal iff $\rho^{*}=\rho$. Hence, for orthogonal representations, Corollary 2.12 implies $T_{M}(\rho)=1$, which agrees with Theorem 2.3 in [RS].

Next consider two closed oriented Riemannian manifolds $M_{i}, i=1,2$, and let $E_{\rho_{i}}$ be a flat bundle over $M_{i}$ defined by a representation $\rho_{i}: \pi_{1}\left(M_{i}\right) \rightarrow$ $G L\left(E_{i}\right)$. We choose a metric $h_{i}$ on $E_{\rho_{i}}$. Furthermore, let

$$
p_{i}: M_{1} \times M_{2} \rightarrow M_{i}, \quad i=1,2,
$$


be the canonical projection. The flat bundle $p_{1}^{*}\left(E_{\rho_{1}}\right) \otimes p_{2}^{*}\left(E_{\rho_{2}}\right)$ over $M_{1} \times M_{2}$ is associated to the representation $\rho_{1} \otimes \rho_{2}$, and we denote by $h_{1} \times h_{2}$ the product metric on this bundle. We also assume that $M_{1} \times M_{2}$ is equipped with the product metric.

Proposition 2.13. With the notation above we have the equality

$$
\begin{aligned}
& \log T_{M_{1} \times M_{2}}\left(\rho_{1} \otimes \rho_{2} ; h_{1} \times h_{2}\right) \\
& \quad=\chi\left(M_{2} ; E_{2}\right) \log T_{M_{1}}\left(\rho_{1} ; h_{1}\right)+\chi\left(M_{1} ; E_{1}\right) \log T_{M_{2}}\left(\rho_{2} ; h_{2}\right),
\end{aligned}
$$

where $\chi\left(M_{i} ; E_{i}\right)$ denotes the Euler characteristic.

This is proved by an easy generalization of the proof of Theorem 2.5 of [RS].

Now assume that the representation $\rho: \pi_{1}(M) \rightarrow \mathrm{GL}(E)$ is unimodular. Then the Reidemeister torsion $\tau_{M}(\rho)$ is defined to be an element of the onedimensional vector space $\left(\operatorname{det} H_{*}(M ; E)\right)^{-1}$. In order to get a real number we have to choose a volume $\mu \in \operatorname{det} H_{*}(M ; E)$. In the present context we choose $\mu$ as follows. Let $h$ be a metric on $E_{\rho}$. Then the inner product on $\Lambda^{*}(E)$ induces an inner product on the space of $E_{\rho}$-valued harmonic forms $\mathscr{H}^{*}(E)$ and we use the De Rham isomorphism (2.1) combined with Poincare duality to introduce an inner product in $H_{*}(M ; E)$. The inner product in $H_{q}(M ; E)$ defines a volume $\mu_{q}$ which, up to sign, is uniquely determined by the metric $h$ on $E_{\rho}$.

Definition 2.14. Let $\mu_{h}=\bigotimes_{q=0}^{n}\left(\mu_{q}\right)^{(-1)^{q}} \in \operatorname{det} H_{*}(M ; E)$ be the volume defined by the metric $h$ on $E$. Then we set

$$
\tau_{M}(\rho ; h)=\tau_{M}\left(\rho ; \mu_{h}\right)
$$

where the latter is the R-torsion of $M$ with respect to $\rho$ and $\mu_{h}$ (cf. $\S 1$ ).

Again the R-torsion depends not only on $h$, but also on $g$, unless $\rho$ is acyclic. The computation of the variational formula for the R-torsion is similar to the orthogonal case and is an easy extension of the proof of Theorem 7.6 in [RS]. The result is

Theorem 2.15. Let $M$ be a closed oriented manifold and $\rho: \pi_{1}(M) \rightarrow \operatorname{GL}(E)$ a unimodular representation. Let $h_{u}$ and $g_{v}$ be one-parameter families of metrics on $E_{\rho}$ and $M$, respectively. Denote by $\tau_{M}(\rho ; u, v)$ the R-torsion and by $P_{q}(u, v)$ the harmonic projection with respect to $\left(h_{u}, g_{v}\right)$. Then

$$
\begin{aligned}
& \frac{\partial}{\partial u} \log \tau_{M}(\rho ; u, v)=\frac{1}{2} \sum_{q=0}^{n}(-1)^{q} \operatorname{Tr}\left(\alpha_{u} P_{q}(u, v)\right), \\
& \frac{\partial}{\partial v} \log \tau_{M}(\rho ; u, v)=\frac{1}{2} \sum_{q=0}^{n}(-1)^{q} \operatorname{Tr}\left(\beta_{v} P_{q}(u, v)\right),
\end{aligned}
$$

where $\alpha_{u}$ and $\beta_{v}$ are defined by (2.3).

If we combine Theorems 2.6 and 2.15, we get 
Corollary 2.16. Let $M$ be a closed oriented manifold, and let $\rho: \pi_{1}(M) \rightarrow$ $\mathrm{GL}(E)$ be a unimodular representation. If $\operatorname{dim} M$ is odd, then

$$
\log T_{M}(\rho ; h)-\log \tau_{M}(\rho ; h)
$$

has the same value for any choice of a metric $h$ on $E_{\rho}$ and a Riemannian metric on $M$. If $\operatorname{dim} M$ is even, then the variation of $\log T_{M}(\rho ; h)-\log \tau_{M}(\rho ; h)$ can be computed as follows. Let $h_{u}$ and $g_{v}$ be one-parameter families of metrics on $E_{\rho}$ and $M$, respectively. Let the notation be the same as in Theorem 2.12. Then

$$
\begin{aligned}
& \frac{\partial}{\partial u}\left(\log T_{M}(\rho ; u, v)-\log \tau_{M}(\rho ; u, v)\right)=\frac{1}{2} \sum_{q=0}^{n}(-1)^{q+1} c_{q}(u, v), \\
& \frac{\partial}{\partial v}\left(\log T_{M}(\rho ; u, v)-\log \tau_{M}(\rho ; u, v)\right)=\frac{1}{2} \sum_{q=0}^{n}(-1)^{q+1} d_{q}(u, v) .
\end{aligned}
$$

The example discussed above shows that for even-dimensional manifolds the variation of $\log T_{M}(\rho ; h)-\log \tau_{M}(\rho ; h)$ may not be zero.

As in the case of orthogonal representations Corollary 2.16 is the key result to prove the equality of $T_{M}(\rho ; h)$ and $\tau_{M}(\rho ; h)$ for closed manifolds of odd dimension. For this purpose it is essential to have the proper generalization of Corollary 2.16 to the case $\partial M \neq \varnothing$. To define the analytic torsion in this case we have to introduce boundary conditions for $\Delta_{q}$. Let $i: \partial M \rightarrow M$ be the inclusion, and let $i^{*}: \Lambda^{*}(E) \rightarrow \Lambda^{*}(E \mid \partial M)$ be the induced map on $E_{\rho}$-valued differential forms. A differential form $\omega \in \Lambda^{*}(E)$ is said to satisfy absolute boundary conditions if

$$
i^{*}(* \omega)=0 \text { and } i^{*}(* d \omega)=0,
$$

and if $\omega$ satisfies absolute boundary conditions then $* \omega$ is said to satisfy relative boundary conditions.

Let $\operatorname{Dom}\left(\Delta_{a}\right)$ (resp. $\left.\operatorname{Dom}\left(\Delta_{r}\right)\right)$ be the subspace of $\Lambda^{*}(E)$ consisting of all those forms which satisfy absolute (resp. relative) boundary conditions on $\partial M$. If $\omega, \theta$ satisfy either boundary conditions, then

$$
\langle d \omega, \theta\rangle=\langle\omega, \delta \theta\rangle
$$

and the restriction of the Laplacian to the corresponding domains defines symmetric positive semidefinite, operators

$$
\Delta_{a}: \operatorname{Dom}\left(\Delta_{a}\right) \longrightarrow \Lambda^{*}(E) \text { and } \Delta_{r}: \operatorname{Dom}\left(\Delta_{r}\right) \longrightarrow \Lambda^{*}(E)
$$

The corresponding selfadjoint extensions on $L^{2} \Lambda^{*}(E)$ have pure point spectrum $0 \leq \lambda_{a, 0} \leq \lambda_{a, 1} \leq \cdots$ and $0 \leq \lambda_{r, 0} \leq \lambda_{r, 1} \leq \cdots$, respectively. Let $\mathscr{H}_{a}^{*}(E)$ and $\mathscr{H}_{r}^{*}(E)$ denote the spaces of harmonic forms for absolute and relative boundary conditions, respectively. Then the De Rham map induces isomorphisms

$$
\mathscr{H}_{a}^{*}(E) \stackrel{\sim}{\longrightarrow} H^{*}(M ; E) \text { and } \mathscr{H}_{r}^{*}(E) \stackrel{\sim}{\longrightarrow} H^{*}(M, \partial M ; E) .
$$

The analytic torsion $T_{M}^{a}(\rho ; h)$ (resp. $\left.T_{M}^{r}(\rho ; h)\right)$ for absolute (resp. relative) boundary conditions is defined by formula (2.2) with $\Delta_{q}$ replaced by $\Delta_{a, q}$ (resp. $\left.\Delta_{r, q}\right)$. 
In order to compute the variation of the analytic torsion we may essentially proceed as in the case of a closed manifold. There are, however, additional complications due to the nonempty boundary.

First we have to study the variation of the trace of the heat operator. We fix absolute boundary conditions. The case of relative boundary conditions follows similarly. Let $h_{u}$ be a one-parameter family of metrics on $E_{\rho}$, and let $\Delta_{a, q}(u)$ denote the Laplacian and $P_{a, q}(u)$ the harmonic projection with respect to $h_{u}$ and the choice of absolute boundary conditions. Let $K_{q}(t ; u)$ denote the kernel of $e^{-t \Delta_{a, q}(u)}$, and let $K_{q}=K_{q}^{\prime}+K_{q}^{\prime \prime}+K_{q}^{\prime \prime \prime}$ denote the Hodge decomposition of $K_{q}$ into its exact, coexact, and harmonic components. To compute the variation of $\operatorname{Tr}\left(e^{-t \Delta_{a, q}(u)}\right)$ we simply follow the proof of Theorem 3.10 in [C] and we note that Duhamel's principle (3.9) which is used in the course of the proof remains valid in our case. As above, let $\alpha=\#^{-1} \#$. Then

$$
\begin{aligned}
\frac{\partial}{\partial u} \operatorname{Tr}\left(e^{-t \Delta_{a, q}(u)}\right)=t \frac{\partial}{\partial t}\{ & \operatorname{Tr}\left(\alpha K_{q+1}^{\prime}(t ; u)\right)-\operatorname{Tr}\left(\alpha K_{q}^{\prime \prime}(t ; u)\right) \\
& \left.+\operatorname{Tr}\left(\alpha K_{q}^{\prime}(t ; u)\right)-\operatorname{Tr}\left(\alpha K_{q-1}^{\prime \prime}(t ; u)\right)\right\} .
\end{aligned}
$$

Applying (2.18), it follows that

$$
\begin{aligned}
& \frac{\partial}{\partial u} \sum_{q=0}^{n}(-1)^{q} q \operatorname{Tr}\left(e^{-t \Delta_{a, q}(u)}-P_{a, q}(u)\right) \\
& \quad=t \frac{\partial}{\partial t} \sum_{q=0}^{n}(-1)^{q} \operatorname{Tr}\left(\alpha\left(e^{-t \Delta_{a, q}(u)}-P_{a, q}(u)\right)\right) .
\end{aligned}
$$

The asymptotic expansion of $\operatorname{Tr}\left(\alpha e^{-t \Delta_{a, q}(u)}\right)$ now contains additional terms coming from the boundary

$$
\operatorname{Tr}\left(\alpha e^{-t \Delta_{a, q}(u)}\right) \sim \sum_{k=0}^{\infty} c_{q, k}^{a}(u) t^{-n / 2+k}+\sum_{k=0}^{\infty} b_{q, k}^{a}(u) t^{-n / 2+k / 2} .
$$

Now we can proceed in exactly the same way as in the closed case, and we get

$$
\frac{\partial}{\partial u} \log T_{M}^{a}\left(\rho ; h_{u}\right)=\frac{1}{2} \sum_{q=0}^{n}(-1)^{q}\left\{\operatorname{Tr}\left(\alpha P_{a, q}(u)\right)-b_{q, n}^{a}(u)\right\}
$$

if $\operatorname{dim} M$ is odd.

A similar relation holds for the variation with respect to the Riemannian metric. Then $\alpha$ has to be replaced by $\beta=*_{v}^{-1} \dot{*}_{v}$ and the coefficients $b_{q, n}^{a}(u)$ by $\tilde{b}_{q, n}^{a}(v)$ occurring in the corresponding asymptotic expansion of $\operatorname{Tr}\left(\beta e^{-t \Delta_{a, q}(u)}\right)$.

To define the R-torsion $\tau_{M}^{a}(\rho ; h)$ we employ the De Rham isomorphism to introduce a volume element for $H^{*}(M ; E)$. The computation of the variation of $\log \tau_{M}^{a}\left(\rho ; h_{u}\right)$ remains the same

$$
\frac{\partial}{\partial u} \log \tau_{M}^{a}\left(\rho ; h_{u}\right)=\frac{1}{2} \sum_{q=0}^{n}(-1)^{q} \operatorname{Tr}\left(\alpha P_{a, q}(u)\right)
$$


Putting (2.20) and (2.21) together we obtain

Theorem 2.22. Let $M$ be a closed manifold of odd dimension and $E_{\rho}$ a flat vector bundle over $M$. Impose absolute boundary conditions. Let $h_{u}$ and $g_{v}$ be one-parameter families of metrics on $E_{\rho}$ and $M$, respectively. Denote by $T_{M}^{a}(\rho ; u, v)$ the analytic torsion and by $\tau_{M}^{a}(\rho ; u, v)$ the R-torsion with respect to $\left(h_{u}, g_{v}\right)$. Then

$$
\begin{gathered}
\frac{\partial}{\partial u}\left(\log T_{M}^{a}(\rho ; u, v)-\log \tau_{M}^{a}(\rho ; u, v)\right)=\frac{1}{2} \sum_{q=0}^{n}(-1)^{q+1} b_{q, n}^{a}(u, v), \\
\frac{\partial}{\partial v}\left(\log T_{M}^{a}(\rho ; u, v)-\log \tau_{M}^{a}(\rho ; u, v)\right)=\frac{1}{2} \sum_{q=0}^{n}(-1)^{q+1} \tilde{b}_{q, n}^{a}(u, v)
\end{gathered}
$$

where the coefficients $b_{q, n}^{a}$ and $\tilde{b}_{q, n}^{a}$ are determined by the asymptotic expansion (2.19) and its counterpart for the variation with respect to $g_{v}$. A similar result holds for relative boundary conditions.

An immediate consequence of Theorem 2.22 is

Corollary 2.23. Let the assumption and notation be the same as in Theorem 2.22. Then for any values $\left(u_{2}, v_{2}\right)$ and $\left(u_{1}, v_{1}\right)$ of the parameters the difference

$\left(\log T_{M}^{a}\left(\rho ; u_{2}, v_{2}\right)-\log \tau_{M}^{a}\left(\rho ; u_{2}, v_{2}\right)\right)-\left(\log T_{M}^{a}\left(\rho ; u_{1}, v_{1}\right)-\log \tau_{M}^{a}\left(\rho ; u_{1}, v_{1}\right)\right)$ depends only on the germs of $h_{u_{2}}, h_{u_{1}}, g_{v_{2}}, g_{v_{1}}$ restricted to $\partial M$ and is completely independent of the geometry and topology of $\operatorname{int}(M)$. The same holds for relative boundary conditions.

Proof. Set

$$
f(u, v)=\log T_{M}^{a}(\rho ; u, v)-\log \tau_{M}^{a}(\rho ; u, v) .
$$

Then, by Theorem 2.22 ,

$$
\begin{aligned}
f\left(u_{2},\right. & \left.v_{2}\right)-f\left(u_{1}, v_{1}\right) \\
& =\int_{u_{1}}^{u_{2}} \frac{\partial}{\partial u} f\left(u, v_{2}\right) d u+\int_{v_{1}}^{v_{2}} \frac{\partial}{\partial v} f\left(u_{1}, v\right) d v \\
& =\frac{1}{2} \sum_{q=0}^{n}(-1)^{q+1}\left\{\int_{u_{1}}^{u_{2}} b_{q, n}^{a}\left(u, v_{2}\right) d u+\int_{v_{1}}^{v_{2}} \tilde{b}_{q, n}^{a}\left(u_{1}, v\right) d v\right\} .
\end{aligned}
$$

By the construction of the asymptotic expansion (2.19) and its analogue for $\operatorname{Tr}\left(\beta e^{-t \Delta_{a, q}(v)}\right)$ the coefficients $b_{q, n}^{a}$ and $\tilde{b}_{q, n}^{a}$ depend only on the germs of $h_{u_{2}}, h_{u_{1}}, g_{v_{2}}, g_{v_{1}}$ restricted to $\partial M$. Q.E.D.

\section{THE EQUALITY OF ANALYTIC TORSION AND R-TORSION}

Let $M$ be a closed oriented Riemannian manifold of odd dimension, and let $\rho: \pi_{1}(M) \rightarrow \mathrm{GL}(E)$ be a unimodular representation with associated flat bundle $E_{\rho}$. Let $h$ be an Euclidean metric on $E_{\rho}$. To establish the equality of the analytic torsion $T_{M}(\rho ; h)$ and the R-torsion $\tau_{M}(\rho ; h)$ we shall follow 
Cheeger's proof for the case of orthogonal representations [C]. As explained in the introduction, this proof is well suited for this purpose because the relevant analysis is done locally near a given handle and this can easily be adapted to the case of unimodular representations.

We recall the basic steps in Cheeger's proof and indicate how they have to be modified (if at all). By Corollary 2.16, $\log T_{M}(\rho ; h)-\log \tau_{M}(\rho ; h)$ is independent of the choice of $h$ and of the metric on $M$. Set

$$
e_{M}(\rho)=\log T_{M}(\rho ; h)-\log \tau_{M}(\rho ; h) .
$$

Furthermore, both $T_{M}(\rho ; h)$ and $\tau_{M}(\rho ; h)$ are independent of the choice of orientation.

Let $M_{0}$ and $M_{1}$ be two closed smooth manifolds of odd dimension $n$, and assume that $M_{1}$ is obtained from $M_{0}$ by surgery on some embedded $k$ sphere $S^{k} \subset M_{0}$ (see below for the precise definition of this statement). Let $E_{0}$ be a flat bundle over $M_{0}$ defined by a unimodular representation $\rho_{0}$ of $\pi_{1}\left(M_{0}\right)$, and assume that $E_{0}$ extends over the trace of the surgery to a flat bundle $E_{1}$ over $M_{1}$ defined by a unimodular representation $\rho_{1}$ of $\pi_{1}\left(M_{1}\right)$. By an argument similar to the one used in the introduction of [C], it suffices to show that $e_{M_{0}}\left(\rho_{0}\right)=0$ implies $e_{M_{1}}\left(\rho_{1}\right)=0$ for all such pairs $\left(M_{0}, \rho_{0}\right)$, $\left(M_{1}, \rho_{1}\right)$. A slight complication arises because this can be proved directly only for $0<k<n-1$.

Now recall that surgery on an embedded $k$-sphere $S^{k} \subset M_{0}$ means the following:

(a) The tubular neighborhood $N\left(S^{k}\right)$ is a product

$$
N\left(S^{k}\right)=S^{k} \times D^{n-k} \text {. }
$$

(b) There is an embedded $(n-k-1)$-sphere $S^{n-k-1} \subset M_{1}$ whose tubular neighborhood is also a product

$$
N\left(S^{n-k-1}\right)=S^{n-k-1} \times D^{k+1}
$$

(c) There is a manifold $\widetilde{M}$ with boundary $\partial \widetilde{M}=S^{k} \times S^{n-k-1}$ such that

$$
M_{0}=N\left(S^{k}\right) \underset{\partial \widetilde{M}}{\cup} \widetilde{M} \text { and } M_{1}=N\left(S^{n-k-1}\right) \underset{\partial \widetilde{M}}{\cup} \widetilde{M}
$$

where the union means that the common boundaries of $\widetilde{M}, N\left(S^{k}\right)$, and $N\left(S^{n-k-1}\right)$ are identified with the obvious identification and the manifolds are given the standard differentiable structures.

The fact that $E_{0}$ extends over the trace of the surgery to give $E_{1}$ is equivalent to the existence of a flat unimodular bundle $\widetilde{E}$ over $\widetilde{M}$ which extends as a flat unimodular bundle over $M_{0}=N\left(S^{k}\right) \cup \widetilde{M}$ and $M_{1}=N\left(S^{n-k-1}\right) \cup \widetilde{M}$. Recall that a flat bundle over a contractible space is trivial as a flat bundle. Since $S^{k} \times\{p\} \subset \partial \widetilde{M}$ bounds $D^{k+1} \times\{p\}$, it follows that $E_{0} \mid S^{k}, S^{k} \subset M_{0}$, and therefore $E_{0} \mid N\left(S^{k}\right)$ is trivial as a flat bundle. Similarly, $E_{1} \mid N\left(S^{n-k-1}\right)$ is trivial as a flat bundle. This is the important fact which allows us to extend Cheeger's analysis. 
We shall employ the following notation. If $X$ is a Riemannian manifold and $Y \subset X$ a submanifold then we shall denote by $N_{u}(Y)$ the tubular neighborhood of $Y$ in $X$ consisting of all normal vectors to $Y$ of length $\leq u$.

Now we introduce metrics on $M_{0}$ and $M_{1}$ such that the tubular neighborhoods $N_{1}\left(S^{k}\right)$ and $N_{1}\left(S^{n-k-1}\right)$ are isometrically the product of the unit spheres $S^{k}, S^{n-k-1}$ and the unit balls $D^{n-k}, D^{k+1}$ equipped with the standard flat metrics. Furthermore, since $E_{0} \mid N_{1}\left(S^{k}\right)$ and $E_{1} \mid N_{1}\left(S^{n-k-1}\right)$ are trivial, we may fix trivializations

$$
E_{0} \mid N_{1}\left(S^{k}\right) \cong N_{1}\left(S^{k}\right) \times \mathbf{R}^{N} \quad \text { and } \quad E_{1} \mid N_{1}\left(S^{n-k-1}\right) \cong N_{1}\left(S^{n-k-1}\right) \times \mathbf{R}^{N}
$$

and choose metrics $h_{0}, h_{1}$ on $E_{0}, E_{1}$ in such a way that they coincide on $E_{0} \mid N_{1}\left(S^{k}\right)$ and $E_{1} \mid N_{1}\left(S^{n-k-1}\right)$ with the product metrics given by the trivializations (3.2). Set

$$
\begin{gathered}
M_{u}= \begin{cases}M_{0}-N_{u}\left(S^{k}\right) & \text { if } 0<u \leq 1 / 3, \\
M_{1}-N_{1-u}\left(S^{n-k-1}\right) & \text { if } 2 / 3 \leq u<1 ;\end{cases} \\
E_{u}= \begin{cases}E_{0} \mid M_{u} & \text { if } 0<u \leq 1 / 3, \\
E_{1} \mid M_{u} & \text { if } 2 / 3 \leq u<1 .\end{cases}
\end{gathered}
$$

We may think of $M_{u}$ (resp. $E_{u}$ ) as being $\widetilde{M}$ (resp. $\widetilde{E}$ ) equipped with the metric $g_{u}$ (resp. $h_{u}$ ). For $1 / 3 \leq u \leq 2 / 3$ let $g_{u}$ (resp. $h_{u}$ ) be any smooth family of metrics connecting these two families but subject to the condition that, near $\partial \widetilde{M}=S^{k} \times S^{n-k-1}, g_{u}$ (resp. $h_{u}$ ) is fixed independent of $M_{0}$, $M_{1}$. As above, we denote by $M_{u}$ (resp. $\left.E_{u}\right), 0<u<1$, the manifold $\widetilde{M}$ (resp. the flat bundle $\widetilde{E}$ ) equipped with $g_{u}$ (resp. $h_{u}$ ).

The structure of the proof of the equality of the two torsions is now the same as in [C]. Let $\rho: \pi_{1}(\widetilde{M}) \rightarrow \mathrm{GL}(E)$ be the unimodular representation that defines $\widetilde{E}$ and set

$$
e_{u}= \begin{cases}\log T_{M}\left(\rho ; h_{u}\right)-\log \tau_{M}\left(\rho ; h_{u}\right) & \text { if } 0<u<1 \\ \log T_{M_{i}}\left(\rho_{i} ; h_{i}\right)-\log \tau_{M_{i}}\left(\rho_{i} ; h_{i}\right) & \text { if } u=i \in\{0,1\}\end{cases}
$$

Then

$$
e_{1}-e_{0}=\left(e_{1}-e_{1-u}\right)+\left(e_{1-u}-e_{u}\right)+\left(e_{u}-e_{0}\right)=A_{u}+B_{u}+C_{u} .
$$

Let $M_{0}^{\prime}, M_{1}^{\prime}, E_{0}^{\prime}, E_{1}^{\prime}$ be a similar set of data where $M_{1}^{\prime}$ is again obtained from $M_{0}^{\prime}$ by surgery on a k-sphere $S^{k}$ (with the same $k$ ). Then

$$
e_{1}^{\prime}-e_{0}^{\prime}=A_{u}^{\prime}+B_{u}^{\prime}+C_{u}^{\prime}
$$

with the obvious notation. By Corollary 2.23, we have $B_{u}=B_{u}^{\prime}$ and the core of the proof is

Theorem 3.3. Let $M_{0}, M_{1}, E_{0}, E_{1}$ and $M_{0}^{\prime}, M_{1}^{\prime}, E_{0}^{\prime}, E_{1}^{\prime}$ be quatruples as above. Then, if $0<k \leq n-1, \lim _{u \rightarrow 0}\left(A_{u}-A_{u}^{\prime}\right)=0$ and, if $0 \leq k<$ $n-1, \lim _{u \rightarrow 0}\left(C_{u}-C_{u}^{\prime}\right)=0$. 
By symmetry, it suffices to consider $C_{u}$. To prove the Theorem for $C_{u}$, we first consider

$$
\left(\log T_{M_{u}}\left(\rho ; h_{u}\right)-\log T_{M_{0}}\left(\rho_{0} ; h_{0}\right)\right)-\left(\log T_{M_{u}^{\prime}}\left(\rho^{\prime} ; h_{u}^{\prime}\right)-\log T_{M_{0}^{\prime}}\left(\rho_{0}^{\prime} ; h_{0}^{\prime}\right)\right)
$$

and investigate its behaviour as $u \rightarrow 0$. Let $\Delta_{q}(u)$ be the Laplacian on $E_{u}$ valued q- forms of $M_{u}, 1>u>0$, satisfying absolute boundary conditions. Let $\zeta_{q}(s ; u)$ be the zeta function associated to $\Delta_{q}(u)$. Then

$$
\log T_{M_{u}}\left(\rho ; h_{u}\right)=\left.\frac{1}{2} \sum_{q=0}^{n}(-1)^{q} q \frac{\partial}{\partial s} \zeta_{q}(s ; u)\right|_{s=0}
$$

and we have to study the behaviour of $\left.\frac{\partial}{\partial s} \zeta_{q}(s ; u)\right|_{s=0}$ as $u \rightarrow 0$.

Recall that there exists an asymptotic expansion

$$
\operatorname{Tr}\left(e^{-t \Delta_{q}(u)}\right)=\sum_{j=0}^{m} a_{q, j}(u) t^{-n / 2+j}+\sum_{j=0}^{2 m} b_{q, j}(u) t^{-n / 2+j / 2}+O\left(t^{-n / 2+m+1 / 2}\right)
$$

as $t \rightarrow 0$. The coefficients $a_{q, j}(u)$ are locally computable in the sense that they are obtained by integrating local densities which in any coordinate system depend in a universal fashion on the coefficients $g_{i, j}(x)$ and $h_{i, j}(x)$ of the metrics and a finite number of their partial derivatives. In the same sense, the coefficients $b_{q, j}(u)$ are locally computable in terms of the extrinsic geometry of the boundary, e.g., its induced metric and second fundamental form (cf. [G] for details). Set

$$
c_{q, j}(u)= \begin{cases}b_{q, j}(u) & \text { if } j=2 l+1, \\ a_{q, j / 2}(u)+b_{q, j}(u) & \text { if } j=2 l .\end{cases}
$$

Furthermore, let $m>n / 2$ and denote by $\mu_{u}(t)$ the difference of $\operatorname{Tr}\left(e^{-t \Delta_{q}(u)}\right)$ and its asymptotic expansion up to order $m$. Denote by $P_{q}(u)$ the harmonic projection. Then, using the definition of $\zeta_{q}(s ; u)$ in terms of the heat kernel, we get

$$
\begin{aligned}
\left.\frac{\partial}{\partial s} \zeta_{q}(s ; u)\right|_{s=0}= & \int_{1}^{\infty} t^{-1}\left(\operatorname{Tr}\left(e^{-t \Delta_{q}(u)}\right)-P_{q}(u)\right) d t+\int_{0}^{1} t^{-1} \mu_{u}(t) d t \\
& +\sum_{j \neq n} \frac{c_{q, j}(u)}{-n / 2+j / 2}+\left(c_{q, n}(u)-\operatorname{dim} H^{q}\left(M_{u} ; E_{u}\right)\right) \gamma
\end{aligned}
$$

where $\gamma=-\Gamma^{\prime}(1)$ is Euler's constant. Now we have to investigate the behaviour of the individual terms as $u \rightarrow 0$. Let $\Delta_{q}$ be the Laplacian on $E_{0}$-valued qforms on $M_{0}$. Then we have a corresponding asymptotic expansion as $t \rightarrow 0$ of the form

$$
\operatorname{Tr}\left(e^{-t \Delta_{q}}\right) \sim \sum_{j=0}^{m} a_{q, j} t^{-n / 2+j}+O\left(t^{-n / 2+m+1}\right) .
$$

The nature of the coefficients $a_{q, j}(u)$ and $a_{q, j}$ implies immediately that

$$
\lim _{u \rightarrow 0} a_{q, j}(u)=a_{q, j}, \quad q=0, \ldots, m .
$$


Furthermore, by assumption, the boundary terms occurring in (3.5) are the same for both $M_{u}$ and $M_{u}^{\prime}$. Hence the contribution to (3.4) made by the coefficients of the asymptotic expansion (3.5) and its analogues for $M_{u}^{\prime}, M_{0}$, and $M_{0}^{\prime}$ will cancel out in the limit.

Next consider $\operatorname{dim} H^{q}\left(M_{u} ; E_{u}\right)$. Recall that $M_{u} \subset M_{0}$ and $E_{u}=E_{0} \mid M_{u}$. Hence there is the long exact cohomology sequence

$$
\begin{aligned}
\cdots & \rightarrow H^{q}\left(M_{0}, M_{u} ; E_{0}\right) \stackrel{j}{\longrightarrow} H^{q}\left(M_{0} ; E_{0}\right) \\
& \stackrel{l}{\longrightarrow} H^{q}\left(M_{u} ; E_{u}\right) \stackrel{\delta}{\longrightarrow} H^{q+1}\left(M_{0}, M_{u} ; E_{0}\right) \rightarrow \cdots
\end{aligned}
$$

and, by excision,

$$
H^{q}\left(M_{0}, M_{u} ; E_{0}\right) \cong H^{q}\left(N\left(S^{k}\right), \partial N\left(S^{k}\right) ; E_{0} \mid N\left(S^{k}\right)\right) .
$$

Using (3.2), we obtain

$$
H^{q}\left(M_{0}, M_{u} ; E_{0}\right) \cong H^{q}\left(N\left(S^{k}\right), \partial N\left(S^{k}\right)\right) \otimes \mathbf{R}^{N} .
$$

Since $M_{1}^{\prime}$ is obtained from $M_{0}^{\prime}$ by surgery on an embedded k-sphere $S^{k} \subset M_{0}$ with the same $k,(3.8)$ also holds for $\left(M_{0}^{\prime}, M_{u}^{\prime}\right)$. Let $k_{q}=\operatorname{dim}\left(\operatorname{ker} l_{q}\right)$. Then, by the exactness of (3.7), we get

$$
\operatorname{dim} H^{q}\left(M_{u} ; E_{u}\right)=\operatorname{dim} H^{q}\left(M_{0} ; E_{0}\right)-k_{q}+\operatorname{dim} H^{q+1}\left(M_{0}, M_{u} ; E_{0}\right)-k_{q+1} .
$$

Let $k_{q}^{\prime}$ have the same meaning with respect to $\left(M_{0}^{\prime}, M_{u}^{\prime}\right)$. Then the contribution to (3.4) of the last term in (3.6) and its analogues for $M_{u}^{\prime}, M_{0}, M_{0}^{\prime}$ is

$$
\gamma \sum_{q=0}^{n}(-1)^{q} q\left(\left(k_{q}+k_{q+1}\right)-\left(k_{q}^{\prime}+k_{q+1}^{\prime}\right)\right) .
$$

This term will be further discussed below.

The essential part is to study the behaviour, as $u \rightarrow 0$, of the first integral on the right-hand side of (3.6). For this purpose we need two estimates describing the behaviour of the heat kernel as $u \rightarrow 0$. Let $K_{u}(x, y, t)$ and $K_{0}(x, y, t)$ denote the kernel of $e^{-t \Delta_{q}(u)}$ and $e^{-t \Delta_{q}}$, respectively.

Theorem 3.11. Let $k<n-1$. Given $T, m, u_{0}>0$, there exists $C_{m}\left(T, u_{0}\right)$ such that, for $t \leq T$ and $x, y \in M_{u_{0}} \subset M_{u}$,

$$
\left\|K_{0}(x, y, t)-K_{u}(x, y, t)\right\| \leq C_{m}\left(T, u_{0}\right) t^{m} \begin{cases}u^{n-k-2} & \text { if } n-k>2, \\ \frac{1}{|\log u|}, & \text { if } n-k=2 .\end{cases}
$$

This theorem states that away from $S^{k} \subset M_{0}$, the heat kernel of $\left(M_{u}, E_{u}\right)$ converges uniformly to that of $\left(M_{0}, E_{0}\right)$ as $u \rightarrow 0$.

The next result describes the behaviour of the heat kernel near $S^{k}$. For $u<u_{0}$, let $A_{u, u_{0}}^{n-k}$ denote the annulus obtained by removing the $(n-k)$-ball $D_{u}^{n-k}$ of radius $u$ from the $(n-k)$-ball $D_{u_{0}}^{n-k}$ of radius $u_{0}$. Let $K_{u, 1}(t)$ be 
the heat kernel on $q$-forms of $S^{k} \times A_{u, 1}^{n-k}$ with coefficients in the trivial flat $\mathbf{R}^{N}$ bundle satisfying absolute boundary conditions. Introduce polar coordinates on $A_{u, 1}^{n-k}$ and write $z \in S^{k} \times A_{u, 1}^{n-k}$ as $z=(x, r)$ where $x \in S^{k} \times S^{n-k-1}$ and $r \in(u, 1)$. Fix $u_{0}<1$.

Theorem 3.12. Let $k<n-1$. Given $T, m$, there exists a constant $C_{m}(T)$ such that

(1) For $r_{1}, r_{2}<u_{0}, x_{1}, x_{2} \in S^{k} \times S^{n-k-1}$,

$$
\begin{aligned}
& \left\|K_{u, 1}\left(\left(x_{1}, r_{1}\right),\left(x_{2}, r_{2}\right), t\right)-K_{u}\left(\left(x_{1}, r_{1}\right),\left(x_{2}, r_{2}\right), t\right)\right\| \\
& \leq C_{m}(T) t^{m}\left\{\begin{array}{cc}
\left(1+u^{n-k-2} r_{1}^{-(n-k-1)}\right)\left(1+u^{n-k-2} r_{2}^{-(n-k-1)}\right) \\
\left(1+\frac{r_{1}^{-1}}{|\log u|}\right)\left(1+\frac{r_{2}^{-1}}{|\log u|}\right), & \text { if } n-k>2, k=2 .
\end{array}\right.
\end{aligned}
$$

(2)

$$
\begin{gathered}
\left|\int_{S^{k} \times A_{u, u_{0}}^{n-k}}\left\{\operatorname{tr}\left(K_{u, 1}((x, r),(x, r), t)\right)-\operatorname{tr}\left(K_{u}((x, r),(x, r), t)\right)\right\}\right| \\
\leq C_{m}(T) t^{m} \begin{cases}\left(u_{0}^{n-k-1}+u^{n-k-3}\right)\left(u_{0}-u\right), & \text { if } n-k>2, \\
u_{0}\left(u_{0}-u\right)+\frac{\log \left(u_{0} / u\right)}{\log ^{2} u}, & \text { if } n-k=2 .\end{cases}
\end{gathered}
$$

The proof of Theorem 3.11 and Theorem 3.12, which, for orthogonal flat bundles, is given in $\S 7$ of [C], depends on two kinds of results. First of all, some standard estimates for the heat kernel are used (cf. [C, $\S 5]$ ). These estimates are derived from Duhamel's principle together with the Sobolev inequality. Both Duhamel's principle and the Sobolev inequality do not require any special assumption on the bundle $E_{\rho}$, and therefore the estimates obtained by Cheeger in $\S 5$ of $[\mathrm{C}]$ are also valid in our setting. The other part that is important for the proof of Theorems 3.11 and 3.12 is the local analysis near $S^{k}$. One has to construct a parametrix for $\Delta_{q}(u)$ satisfying absolute boundary conditions which allows us to study its behaviour as $u \rightarrow 0$. The parametrix is obtained by patching an interior parametrix and a parametrix near the boundary. The interior parametrix is obtained by general constructions which again do not require special assumptions. To construct the parametrix near the boundary it suffices to construct the Green's operator for $\Delta$ on $\mathbf{R}^{N}$-valued differential forms on $S^{k} \times A_{u, 1}^{n-k}$ satisfying absolute boundary conditions. By our assumption, the metrics on $S^{k} \times A_{u, 1}^{n-k}$ and

$$
E_{u} \mid\left(S^{k} \times A_{u, 1}^{n-k}\right) \cong S^{k} \times A_{u, 1}^{n-k} \times \mathbf{R}^{N}
$$

are the standard product metrics. Therefore, all the local analysis done by Cheeger in [C, $\S \S 6,7]$ can be applied to our case without any change. This suffices to prove Theorems 3.11 and 3.12. We just follow the proof of these theorems in [C, §7] line by line. 
In order to estimate $\operatorname{Tr}\left(e^{-t \Delta_{q}(u)}\right)$ for large $t$ one has to study the small eigenvalues, that is, those eigenvalues of $\Delta_{q}(u)$ which converge to zero as $u \rightarrow 0$. Set

$$
r(q)=\operatorname{dim} H^{q-(n-k-1)}\left(S^{k}\right) .
$$

The behaviour of the small eigenvalues is described by

Proposition 3.13. There exists $\lambda>0$ with the following property. For all $0<$ $\varepsilon<\lambda$, there exists $\delta>0$ such that, for $u<\delta, \Delta_{q}(u)$ has exactly

$$
\operatorname{dim} H^{q}\left(M_{0} ; E_{0}\right)+N \cdot r(q)
$$

eigenvalues $<\lambda$ and all of them are $<\varepsilon$.

The proof is exactly the same as the proof of Proposition 7.19 in [C].

Let $0<\lambda_{1}^{q}(u) \leq \lambda_{2}^{q}(u) \leq \cdots \leq \lambda_{s(q)}^{q}(u)<\lambda$ be the nonzero eigenvalues described by Proposition 3.13 . By Proposition 3.13, we have

$$
\operatorname{dim} H^{q}\left(M_{u} ; E_{u}\right)+s(q)=\operatorname{dim} H^{q}\left(M_{0} ; E_{0}\right)+\operatorname{dim} H^{q+1}\left(M_{0}, M_{u} ; E_{0}\right) .
$$

Comparing this with (3.9), we get

$$
k_{q}+k_{q+1}=s(q) \text {. }
$$

Now let $\Delta_{q}^{\prime}(u)$ denote the restriction of $\Delta_{q}(u)$ to the orthogonal complement in $L^{2} \Lambda^{q}\left(E_{u}\right)$ of the sum of the eigenspaces corresponding to the small eigenvalues $<\lambda$ described by Proposition 3.13. Note that $\lambda^{-s}=\frac{1}{\Gamma(s)} \int_{0}^{\infty} t^{s-1} e^{-\lambda t} d t$. Taking the derivative at $s=0$ gives

$$
\int_{1}^{\infty} t^{-1} e^{-\lambda t} d t=-\log \lambda-\int_{0}^{1} t^{-1}\left(e^{-\lambda t}-1\right) d t-\gamma
$$

where $\gamma=-\Gamma^{\prime}(1)$. Then the first integral on the right-hand side of (3.6) equals $-\sum_{j=1}^{s(q)} \log \lambda_{j}^{q}(u)+\int_{1}^{\infty} t^{-1} \operatorname{Tr}\left(e^{-t \Delta_{q}^{\prime}(u)}\right) d t-\int_{0}^{1} t^{-1}\left(\sum_{j=1}^{s(q)} e^{-\lambda_{j}^{q}(u) t}-s(q)\right) d t-s(q) \gamma$.

By (3.14), the contribution of the last term to (3.4) cancels (3.10). Furthermore, by Proposition 3.13 , the second integral tends to zero as $u \rightarrow 0$, and for any given $\varepsilon>0$ there exists $T^{\prime}$ such that, for $T>T^{\prime}, u<\delta$,

$$
\int_{T}^{\infty} t^{-1} \operatorname{Tr}\left(e^{-t \Delta_{q}^{\prime}(u)}\right) d t<\varepsilon
$$

Fix $u_{0}, T>0$. Then

$$
\begin{aligned}
\int_{1}^{T} t^{-1} \operatorname{Tr}\left(e^{-t \Delta_{q}^{\prime}(u)}\right) d t= & \int_{1}^{T} t^{-1} \int_{M_{u_{0}}} \operatorname{tr} K_{u}^{q}(x, x, t) d x d t \\
& +\int_{1}^{T} t^{-1} \int_{S^{k} \times A_{u, u_{0}}^{n-k}} \operatorname{tr} K_{u}^{q}(x, x, t) d x d t \\
& -\sum_{j=1}^{s(q)} \int_{1}^{T} t^{-1} e^{-\lambda_{j}^{q}(u) t} d t-\log T \operatorname{dim} H^{q}\left(M_{u} ; E_{u}\right) .
\end{aligned}
$$


By Proposition 3.13, the last two terms tend to

$$
-\log T\left(s(q)+\operatorname{dim} H^{q}\left(M_{u} ; E_{u}\right)\right)
$$

as $u \rightarrow 0$, and, by Proposition 3.13, this equals

$$
-\log T\left(\operatorname{dim} H^{q}\left(M_{0} ; E_{0}\right)+N \cdot r(q)\right) .
$$

The same holds if we replace $M_{u}$ by $M_{u}^{\prime}$ so that the combined contribution to (3.3) of the last two terms in (3.15) and the analogous terms with respect to $M_{u}^{\prime}$ will cancel out in the limit $u \rightarrow 0$.

Finally, one uses Theorems 3.11 and 3.12 to tread the remaining integrals. Let $\underline{K}_{u, 1}(t)$ denote the kernel obtained by projecting the heat kernel $K_{u, 1}(t)$ defined above onto the orthogonal complement of the harmonic forms. Then the final result can be stated as

$$
\begin{aligned}
\lim _{u_{0} \rightarrow 0} \lim _{u \rightarrow 0} \mid & \int_{1}^{\infty} t^{-1} \operatorname{Tr}\left(e^{-t \Delta_{q}(u)}-P^{q}(u)\right) d t-\int_{1}^{\infty} t^{-1} \operatorname{Tr}\left(e^{-t \Delta_{q}}-P^{q}\right) d t \\
& -\int_{1}^{\infty} t^{-1} \int_{S^{k} \times A_{u, u_{0}}^{n-k}} \operatorname{Tr}\left(\underline{K}_{u, 1}(x, x, t)\right) d x d t+\sum_{j=1}^{s(q)} \log \left(\lambda_{j}^{q}(u)\right) \mid=0 .
\end{aligned}
$$

Next we have to investigate the integrals $\int_{0}^{1} t^{-1} \mu_{u}(t) d t$ and $\int_{0}^{1} t^{-1} \mu_{0}(t) d t$. Recall that $\mu_{u}(x, t), \mu_{0}(x, t)$, and $\mu_{u, 1}(x, t)$ are the differences between $\operatorname{tr} K_{u}^{q}(x, x, t), \operatorname{tr} K_{0}^{q}(x, x, t)$, and $\operatorname{tr} K_{u, 1}^{q}(x, x, t)$, respectively, and their asymptotic expansion up to order $m>n / 2$. Then the argument which led to $(3.16)$ can also be used to prove

$$
\begin{aligned}
\lim _{u_{0} \rightarrow 0} \lim _{u \rightarrow 0} \mid \int_{0}^{1} t^{-1} \mu_{u}(t) & d t-\int_{0}^{1} t^{-1} \int_{M_{0}} \mu_{0}(x, t) d x d t \\
& -\int_{0}^{1} t^{-1} \int_{S^{k} \times A_{u, u_{0}}^{n-k}} \mu_{u, 1}(x, t) d x d t \mid=0 .
\end{aligned}
$$

Now consider $M_{0}^{\prime}, M_{u}^{\prime}, E_{0}^{\prime}, E_{u}^{\prime}$. Let $0<\lambda_{1}^{\prime q}(u) \leq \cdots \leq \lambda_{s(q)}^{\prime q}(u)<\lambda$ be the nonzero eigenvalues described by Proposition 3.13 with respect to $M_{u}^{\prime}, E_{u}^{\prime}$. Subtracting the corresponding versions of (3.16) and (3.17) for $M_{0}^{\prime}, M_{u}^{\prime}, E_{0}^{\prime}, E_{u}^{\prime}$ from (3.16) and (3.17), respectively, and using (3.6) we can summarize our results by

$$
\begin{aligned}
& \lim _{u \rightarrow 0}\left\{\left(\log T_{M_{u}}\left(\rho ; h_{u}\right)-\log T_{M_{0}}\left(\rho_{0} ; h_{0}\right)\right)\right.-\left(\log T_{M_{u}^{\prime}}\left(\rho^{\prime} ; h_{u}^{\prime}\right)-\log T_{M_{0}^{\prime}}\left(\rho_{0}^{\prime} ; h_{0}^{\prime}\right)\right) \\
&\left.\quad+\sum_{q=0}^{n}(-1)^{q} q \sum_{j=1}^{s(q)} \log \left(\lambda_{j}^{q}(u) / \lambda_{j}^{\prime q}(u)\right)\right\}=0 .
\end{aligned}
$$

It remains to investigate the corresponding expression for the R-torsion. First we consider

$$
\log \tau_{M_{u}}\left(\rho ; h_{u}\right)-\log \tau_{M_{0}}\left(\rho_{0} ; h_{0}\right)
$$


Let $K_{0}$ be a smooth triangulation of $M_{0}$ and $K^{\prime} \subset K_{0}$ a subcomplex which induces a triangulation of the submanifold $\widetilde{M} \subset M_{0}$. Recall that $M_{u}$ denotes the manifold $\widetilde{M}$ equipped with the metric $g_{u}$. Furthermore, $\widetilde{E}=E_{0} \mid \widetilde{M}$ and $E_{u}$ is the flat bundle $\widetilde{E}$ equipped with the metric $h_{u}$. Then we get a short exact sequence

$$
0 \rightarrow C_{\bullet}\left(K^{\prime} ; \widetilde{E}\right) \longrightarrow C_{\bullet}\left(K_{0} ; E_{0}\right) \longrightarrow C_{\bullet}\left(K_{0}, K^{\prime} ; E_{0}\right) \rightarrow 0
$$

of chain complexes. Each of these chain complexes has a distinguished volume determined by preferred bases and the metrics $h_{0}, g_{0}, h_{u}, g_{u}$ determine volumes $\mu_{0} \in \operatorname{det} H_{*}\left(M_{0} ; E_{0}\right), \mu_{u} \in \operatorname{det} H_{*}\left(M_{u} ; E_{u}\right)$. Since $M_{0}-\widetilde{M} \cong N\left(S^{k}\right)$, $E_{0} \mid N\left(S^{k}\right) \cong N\left(S^{k}\right) \times \mathbf{R}^{N}$, and the metrics are the standard product metrics, the torsion of the relative chain complex equals $\tau_{N\left(S^{k}\right)}(\mathbf{1})^{N}$ where $\mathbf{1}$ stands for the trivial one-dimensional representation. Moreover, it is easy to verify that the torsion of each complex

$$
0 \rightarrow C_{q}\left(K^{\prime} ; \widetilde{E}\right) \longrightarrow C_{q}\left(K_{0} ; E_{0}\right) \longrightarrow C_{q}\left(K_{0}, K^{\prime} ; E_{0}\right) \rightarrow 0
$$

equals 1. By Theorem 3.2 in [Mi1], it follows that

$$
\log \tau_{M_{0}}\left(\rho_{0} ; h_{0}\right)=\log \tau_{M_{u}}\left(\rho, h_{u}\right)+N \cdot \log \tau_{N\left(S^{k}\right)}(\mathbf{1})+\log \tau\left(\mathscr{H}_{\bullet} ; \mu_{u}\right)
$$

where $\mathscr{C}_{\bullet}$ denotes the long exact homology sequence of (3.19) regarded as an acyclic chain complex of length $3 n$ equipped with the volumes $\mu_{u}, \mu_{0}$. Subtracting the corresponding equation for $M_{0}^{\prime}, M_{u}^{\prime}, E_{0}^{\prime}, E_{u}^{\prime}$, we get

$$
\begin{aligned}
\lim _{u \rightarrow 0}\left\{\left(\log \tau_{M_{u}}\left(\rho ; h_{u}\right)-\log \tau_{M_{0}}\left(\rho_{0} ; h_{0}\right)\right)\right. \\
-\left(\log \tau_{M_{u}^{\prime}}\left(\rho^{\prime} ; h_{u}^{\prime}\right)-\log \tau_{M_{0}^{\prime}}\left(\rho_{0}^{\prime} ; h_{0}^{\prime}\right)\right) \\
\left.\quad+\left(\log \tau\left(\mathscr{H}_{\bullet} ; \mu_{u}\right)-\log \tau\left(\mathscr{H}_{\bullet}^{\prime} ; \mu_{u}^{\prime}\right)\right)\right\}=0 .
\end{aligned}
$$

Now one has to study the behaviour of $\log \tau\left(\mathscr{H}_{\bullet} ; \mu_{u}\right)$ and $\log \tau\left(\mathscr{H}_{\bullet}^{\prime} ; \mu_{u}^{\prime}\right)$ as $u \rightarrow 0$. This is completely analogous to [C] and we leave it to the reader (cf. $\left[C\right.$, p. 316]). As result we obtain that, in the limit $u \rightarrow 0, \log \tau\left(\mathscr{H}_{\bullet} ; \mu_{u}\right)-$ $\log \tau\left(\mathscr{H}_{\bullet}^{\prime} ; \mu_{u}^{\prime}\right)$ cancels the contribution of the small eigenvalues in (3.18). This completes the proof of Theorem 3.3.

Now we can proceed exactly in the same way as in [C]. Write $d_{k}=e_{1}-e_{0}$ where $e_{i}=\log T_{M_{i}}\left(\rho_{i} ; h_{i}\right)-\log \tau_{M_{i}}\left(\rho_{i} ; h_{i}\right)$ and $M_{1}$ is obtained from $M_{0}$ by surgery on an embedded $k$-sphere.

Proposition 3.21. We have $d_{k}=0$ for $1 \leq k \leq n-2$. Moreover, $d_{0}$ is well defined.

We just follow the proofs of Proposition 8.20 and 8.21 of [C] line by line to obtain the proof of Proposition 3.21.

We can now prove Theorem 1 of the introduction. To prove this theorem we proceed in exactly the same way as on p. 318 of [C], using Propositions 1.12, $1.13,2.9,2.13$. 
Remark. Everything that has been said in the previous sections works also for finite-dimensional complex representations of $\pi_{1}(M)$.

\section{SOME EXAMPLES}

In this section we shall discuss two examples where nonorthogonal or nonunitary representations of the fundamental group arise naturally and the torsion is of relevance in this context

4.1. Locally symmetric manifolds. Let $G$ be a connected semisimple Lie group with finite center. We also assume that $G$ has no compact factors. Let $K$ be a maximal compact subgroup of $G$. The Lie algebras of $G$ and $K$ will be denoted by $\mathfrak{g}$ and $\mathfrak{k}$, respectively. Let $\mathfrak{g}=\mathfrak{k} \oplus \mathfrak{p}$ be the Cartan decomposition of $\mathfrak{g}$ with respect to $\mathfrak{k}$, and let $\theta$ be the Cartan involution of $(\mathfrak{g}, \mathfrak{k})$. The quotient space $X=G / K$ is then a symmetric Riemannian manifold and $G$ is the identity component of the group of orientation preserving isometries of $X$. We shall denote by $x_{0} \in X$ the coset $e K$ of the identity $e \in G$.

Let $\Gamma \subset G$ be a discrete, torsionfree, co-compact subgroup of $G$. Then $M=$ $\Gamma \backslash X$ is a compact locally symmetric manifold covered by $X$ with $\pi_{1}(M)=\Gamma$.

Let $\rho: G \rightarrow \mathrm{GL}(E)$ be a representation of $G$ on a finite-dimensional complex vector space $E$. If we restrict $\rho$ to $\Gamma$, we obtain a representation $\rho_{\Gamma}: \Gamma \rightarrow \mathrm{GL}(E)$ with associated flat vector bundle $E_{\rho}$ over $\Gamma \backslash X$. Since $E$ is a $\Gamma$-module, the group cohomology $H^{*}(\Gamma ; E)$ is defined and we have the equality

$$
H^{*}\left(\Gamma \backslash X ; E_{\rho}\right)=H^{*}(\Gamma ; E) .
$$

There is a different way to describe the bundle $E_{\rho}$. Let $\rho_{K}: K \rightarrow \mathrm{GL}(E)$ be the restriction of $\rho$ to $K$, and consider the fibration

$$
\Gamma \backslash G \longrightarrow \Gamma \backslash X
$$

which is principal with structure group $K$. Then $\rho_{K}$ defines the induced bundle $\widetilde{E}_{\rho}$ over $\Gamma \backslash X$ whose global $C^{\infty}$-sections are the $C^{\infty}$-functions $f: \Gamma \backslash G \longrightarrow E$ which satisfy

$$
f(g k)=\rho(k)^{-1}(f(g)) \text { for all } g \in G, k \in K \text {. }
$$

Lemma 4.2. The bundles $E_{\rho}$ and $\widetilde{E}_{\rho}$ are naturally isomorphic. If $\tilde{f}: \Gamma \backslash G \rightarrow E$ is a section of $\widetilde{E}_{\rho}$, set $f(x)=f\left(g x_{0}\right)=\rho(g) \tilde{f}(g)$ where $x=g x_{0}$. Then $f: X \rightarrow E$ defines a section of $E_{\rho}$ and $\tilde{f} \mapsto f$ establishes an isomorphism of the corresponding spaces of $C^{\infty}$-sections.

For the proof see Proposition 3.1 in [MM].

Now observe that the flat bundles $E_{\rho}$ defined by a representation $\rho$ : $G \rightarrow \mathrm{GL}(E)$ fit into the setting of the previous sections. Namely, we have

Lemma 4.3. Any finite-dimensional representation $\rho: G \rightarrow \mathrm{GL}(E)$ satisfies $\operatorname{det} \rho(g)=1$ for all $g \in G$.

Proof. Let $N=\operatorname{dim}_{\mathrm{C}} E$. Then $G$ acts on $\Lambda^{N} E$ via the character $\chi=\operatorname{det} \circ \rho$. Since $G$ is semisimple, we have $D \mathfrak{g}=\mathfrak{g}$ and, therefore, $d \chi=0$. But $G$ is connected, which implies $\chi \equiv 1$. Q.E.D. 
Thus each of the flat bundles $E_{\rho}$ constructed above is unimodular. Next we equip $E_{\rho}$ with a canonical metric. Following Matsushima and Murakami in [MM], we call a hermitian inner product $\langle u, v\rangle$ on $E$ admissible if

(a) $\langle\rho(Y) u, v\rangle=-\langle u, \rho(Y) v\rangle$ for all $Y \in \mathfrak{k}, u, v \in E$;

(b) $\langle\rho(Y) u, v\rangle=\langle u, \rho(Y) v\rangle$ for all $Y \in \mathfrak{p}, u, v \in E$.

The existence of an admissible inner product on any $G$-module $E$ is proved in [MM, Lemma 3.1]. Condition (a) means that $\langle\cdot, \cdot\rangle$ is invariant under $\rho(K)$, and therefore it defines a hermitian metric on $E_{\rho}$. This is the canonical metric on $E_{\rho}$ defined by the admissible inner product on $E$.

This choice of an inner product on $E_{\rho}$ allows us to use harmonic analysis to study the Laplacian $\Delta$ on $E_{\rho}$-valued differential forms on $\Gamma \backslash X$. The tangent space to $X$ at $x_{0}$ can be identified with $\mathfrak{p}$ and the tangent bundle of $\Gamma \backslash X$ is the bundle induced from (4.1) by the adjoint representation $\operatorname{ad}_{\mathfrak{p}}: K \rightarrow \mathrm{GL}(\mathfrak{p})$. Therefore, we have a natural identification

$$
\begin{aligned}
\Lambda^{q}(\Gamma \backslash X, E)=\{ & \left\{\varphi: \Gamma \backslash G \rightarrow \Lambda^{q} \mathfrak{p}^{*} \otimes E \mid \varphi \text { is } C^{\infty}\right. \text { and } \\
& \left.\varphi(g k)=\left(\Lambda^{q} \operatorname{ad}_{\mathfrak{p}}^{*}\left(k^{-1}\right) \otimes \rho\left(k^{-1}\right)\right)(\varphi(g)), g \in G, k \in K\right\} .
\end{aligned}
$$

Concerning the cohomology $H^{*}(\Gamma ; E)$ there exist plenty of results, in particular, vanishing theorems (cf. [BW, Chapter VII]) telling us which representations are acyclic. We mention one of them. Let $\mathfrak{h}^{+} \subset \mathfrak{k}$ be a maximal abelian subalgebra of $\mathfrak{k}$. Let $\mathfrak{h}$ be the centralizer of $\mathfrak{h}^{+}$in $\mathfrak{g}$. Then $\mathfrak{h}$ is a Cartan subalgebra of $\mathfrak{g}$. Let $\Phi$ be a root system of $\left(\mathfrak{g}_{\mathbf{C}}, \mathfrak{h}_{\mathbf{C}}\right)$, and let $\Phi_{k}$ be a root system of $\left(\mathfrak{k}_{\mathbf{C}}, \mathfrak{h}_{\mathbf{C}}^{+}\right)$. Fix a set $\Phi_{k}^{+}$of positive roots for $\Phi_{k}$, and let $\Phi^{+}$be a system of positive roots for $\Phi$ compatible with $\Phi_{k}^{+}$(see [BW, p. 65]). This means, in particular, that $\theta \alpha \in \Phi^{+}$whenever $\alpha \in \Phi^{+}$. As usually set

$$
2 \underline{\rho}=\sum_{\alpha \in \Phi^{+}} \alpha .
$$

Theorem 4.4 (Borel-Wallach). Let $E$ be an irreducible finite-dimensional $G$ module with highest weight $\Lambda-\underline{\rho}$. If $\theta \Lambda \neq \Lambda$ then $H^{*}(\Gamma ; E)=0$.

For the proof see Theorem 6.7 of Chapter VII in [BW].

Let $\mathfrak{h}_{\mathbf{C}}^{-}=\left\{H \in \mathfrak{h}_{\mathbf{C}} \mid \theta H=-H\right\}$. Then $\mathfrak{h}_{\mathbf{C}}^{*}=\left(\mathfrak{h}_{\mathbf{C}}^{+}\right)^{*} \oplus\left(\mathfrak{h}_{\mathbf{C}}^{-}\right)^{*}$. Moreover, assume that $\operatorname{rk} G>\operatorname{rk} K$. Then $\operatorname{dim} \mathfrak{h}_{\mathbf{C}}^{-} \geq 1$ and the highest weight of a generic representation satisfies $\theta \Lambda \neq \Lambda$. Note that the condition $\operatorname{rk} G>\operatorname{rk} K$ is satisfied whenever $\operatorname{dim} G / K$ is odd, which is the case we are mainly interested in. Thus, for compact locally symmetric manifolds $\Gamma \backslash X$ of odd dimension, Theorem 4.4 produces a large class of acyclic unimodular representations of $\Gamma$.

As an example we shall discuss three-dimensional hyperbolic manifolds. In this case we have $G=\mathrm{SL}(2, \mathrm{C})$ and $K=\mathrm{SU}(2)$, and $H^{3}=\mathrm{SL}(2, \mathrm{C}) / \mathrm{SU}(2)$ is the three-dimensional hyperbolic space. Let $\Gamma \subset \mathrm{SL}(2, \mathrm{C})$ be as above, that is, a discrete, torsionfree, co-compact subgroup. Since $\operatorname{SL}(2, C)$ is simple, $\Gamma$ is an irreducible discrete subgroup.

To describe the irreducible finite-dimensional representations of $\operatorname{SL}(2, C)$, we have to consider $\mathfrak{s l}(2, \mathbf{C})$ as Lie algebra over $\mathbf{R}$ which we denote by 
$\mathfrak{s l}(2, \mathbf{C})^{\mathbf{R}}$. It is well known that

$$
\mathfrak{s l}(2, \mathbf{C})^{\mathbf{R}} \cong \mathfrak{s o}(3,1) \text { and } \mathfrak{s u}(2) \cong \mathfrak{s o}(3, \mathbf{R})
$$

where $\mathfrak{s o}(3,1)$ is the Lie algebra of $\operatorname{SO}(3,1) \subset \mathrm{GL}(4, \mathbf{R})$. Furthermore, there exists an isomorphism

$$
\varphi: \mathfrak{s o}(3,1) \underset{\mathbf{R}}{\otimes} \mathbf{C} \cong \mathfrak{s o}(4, \mathbf{C}) \cong \mathfrak{s l}(2, \mathbf{C}) \times \mathfrak{s l}(2, \mathbf{C})
$$

such that

$$
\varphi(\mathfrak{s o}(3,1))=\{(X, \bar{X}) \mid X \in \mathfrak{s l}(2, \mathbf{C})\} .
$$

Next recall that the irreducible finite-dimensional representations of the complex Lie algebra $\mathfrak{s l}(2, \mathbf{C})$ are parametrized by $p \in \mathbf{N}$. Given $p \in \mathbf{N}$, let $W_{p}$ be the corresponding irreducible $\mathfrak{s l}(2, \mathbf{C})$-module. Then $W_{1}=\mathbf{C}^{2}$ and $\rho_{1}: \mathfrak{s l}(2, \mathbf{C}) \rightarrow \mathfrak{g l}\left(\mathbf{C}^{2}\right)$ is the standard representation. Furthermore, for each $p \in \mathbf{N}, W_{p}$ is the $p$ th symmetric power of the module $W_{1}=\mathbf{C}^{2}$.

Let $E$ be a finite-dimensional complex vector space. Then the complex conjugate $\bar{E}$ of $E$ is defined to be the dual vector space of the vector space of all antilinear forms on $E$. Every $x \in E$ determines a unique element $\bar{x} \in \bar{E}$. In particular, we can consider the complex conjugate $\bar{W}_{q}$ of the $\mathfrak{s l}(2, \mathbf{C})$-module $W_{q}$. Then $\mathfrak{s l}(2, \mathrm{C})$ acts on $\bar{W}_{q}$ by

$$
\bar{\rho}_{q}(X) \bar{w}=\overline{\rho_{q}(X) w}, \quad X \in \mathfrak{s l}(2, \mathbf{C}), \bar{w} \in \bar{W}_{q},
$$

and we get an antiholomorphic representation

$$
\bar{\rho}_{q}: \mathfrak{s l}(2, \mathbf{C}) \longrightarrow \mathfrak{g l}\left(\bar{W}_{q}\right) .
$$

Given $(p, q) \in \mathbf{N}^{2}$, set $\tilde{\rho}_{p, q}=\rho_{p} \otimes \bar{\rho}_{q}$. Then

$$
\tilde{\rho}_{p, q}: \mathfrak{s l}(2, \mathbf{C}) \longrightarrow \mathfrak{g l}\left(W_{p} \otimes \bar{W}_{q}\right)
$$

is an irreducible representation of $\mathfrak{s l}(2, \mathbf{C})^{\mathbf{R}}$. Since $\operatorname{SL}(2, \mathbf{C})$ is simply connected, $\tilde{\rho}_{p, q}$ can be lifted to $\operatorname{SL}(2, \mathbf{C})$. Hence, for each $(p, q) \in \mathbf{N}^{2}$, we get an irreducible representation

$$
\rho_{p, q}: \mathrm{SL}(2, \mathbf{C}) \longrightarrow \mathrm{GL}\left(W_{p} \otimes \bar{W}_{q}\right) ;
$$

any irreducible representation of the real Lie group $\operatorname{SL}(2, C)$ is obtained in this way. This is a special case of a more general result (cf. [Wa, Theorem 3.1.1.1]). With respect to this parametrization, the Cartan involution acts by $\theta(p, q)=(q, p)$. Employing Theorem 4.4, we obtain

Lemma 4.6. Let $\Gamma$ be a discrete, torsionfree, co-compact subgroup of $\mathrm{SL}(2, \mathrm{C})$. For $(p, q) \in \mathbf{N}^{2}$, let $E_{p, q}$ be the irreducible $\operatorname{SL}(2, \mathbf{C})$-module described above. Then

$$
H^{*}\left(\Gamma \backslash H^{3}, E_{p, q}\right)=0 \text { if } p \neq q .
$$

We note that the complexification of the standard representation of $\mathfrak{s o}(3,1)$ on $\mathbf{R}^{4}$ is equivalent to the $\mathfrak{s l}(2, \mathbf{C})$-module $E_{1,1}$. Thus we can restate Lemma 
4.6 as follows. If a given irreducible $\mathfrak{s l}(2, \mathrm{C})$-module $E_{\Lambda}$ with heighest weight $\Lambda$ has nontrivial cohomology, then $\Lambda$ is a multiple of the heighest weight of the standard representation of $\mathfrak{s o}(3,1)$. This agrees with Theorem 1 in [R].

For all these acyclic representations $\rho$, the analytic torsion $T_{M}(\rho)$ and the R-torsion $\tau_{M}(\rho)$ are defined and independent of any choice of metrics. This is of interest in connection with the results of Moscovici and Stanton [MS].

4.2. Chern-Simons gauge theory. Chern-Simons gauge theory is a three-dimensional gauge field theory with pure Chern-Simons action. The connection with analytic torsion was first noticed by Schwarz [S] in the case of an abelian gauge group. For nonabelian gauge groups the link to analytic torsion was established by Witten [W1] via the perturbative expansion of the corresponding functional integral. For a compact gauge group, the torsion invariants occurring in the perturbative expansion are associated to unitary representations of the fundamental group of the underlying 3-manifold. However, if we allow noncompact semisimple gauge groups, then one has to deal with the analytic torsion attached to unimodular representations.

Now we shall describe this in more detail. Let $M$ be a compact oriented three-dimensional manifold without boundary and $G$ a Lie group. We start with the case where $G$ is compact, and for simplicity we take $G$ to be $\operatorname{SU}(N)$. Consider the space $\mathscr{A}$ of all $G$-connections on the trivial $G$-bundle over $M$. In fact, every principal $G$-bundle over $M$ is trivial. The space $\mathscr{A}$ may be identified with the space $\Lambda^{1}(M, \mathfrak{g})$ of differential 1 -forms on $M$ with values in the Lie algebra $\mathrm{g}$ of $G$. For a given connection $A \in \mathscr{A}$, the Chern-Simons action is defined to be

$$
I(A)=\frac{1}{4 \pi} \int_{M} \operatorname{Tr}\left(A \wedge d A+\frac{2}{3} A \wedge A \wedge A\right)
$$

where $\operatorname{Tr}$ is the trace of $\mathfrak{s u}(N)$ in the standard representation. This is a realvalued nonlinear functional on $\mathscr{A}$. The gauge group $\mathscr{G}=\operatorname{Map}(M, G)$ acts on $\mathscr{A}$ by the usual prescription $A^{g}=g^{-1} A g+g^{-1} d g, g \in G, A \in \mathscr{A}$. The Chern-Simons functional $I$ is not invariant under the action of $\mathscr{G}$, but it satisfies $I\left(A^{g}\right)=I(A)+2 \pi m$ for some $m \in \mathbf{Z}$ depending on $g \in \mathscr{G}$. Let $k \in \mathbf{N}$. Then $e^{i k I(A)}$ is a $\mathscr{G}$-invariant function on $\mathscr{A}$ and Witten's invariant of $M$ is defined as the path integral

$$
Z_{M}(k)=\int e^{i k I(A)} \mathscr{D} A
$$

where the integration is over all gauge equivalence classes of connections. This, however, has to be considered as a formal expression because no measure $\mathscr{D} A$ has yet been constructed. Part of this theory can be made rigorous, and Witten gave an explicit recipe for computing $Z_{M}(k)$. Moreover, using the theory of quantum groups, Reshetikhin and Turaev [RT] introduced invariants $\widetilde{Z}_{M}(q)$ of a 3-manifold $M$ depending on a root of unity $q=e^{2 \pi i / r}$, and they suggest that $\widetilde{Z}_{M}\left(e^{2 \pi i /(k+2)}\right)$ coincides with $Z_{M}(k)$ after normalization.

Instead of the full path integral $(4.8)$ one can also study the perturbative formulation, i.e., one perturbs about the critical points $A^{(0)}$ of the action (4.7). 
In the present context this method is again not based on solid ground, but it gives very interesting results. The Euler-Lagrange equation for (4.7) is

$$
d A+A \wedge A=0
$$

which shows that the critical points of (4.7) are precisely the connections with vanishing curvature, that is, the flat connections on the bundle $P=M \times G$. A flat connection $A$ is determined up to gauge equivalence by a representation

$$
\alpha: \pi_{1}(M) \rightarrow G
$$

up to conjugacy. Hence the space of gauge equivalence classes of flat connections on $P$ can be identified with $\operatorname{Hom}\left(\pi_{1}(M), G\right) / G$ where $G$ acts on $\operatorname{Hom}\left(\pi_{1}(M), G\right)$ by conjugation. Assume that the topology of $M$ is such that there exists only a finite number of gauge equivalence classes of flat connections on $P$, say $A_{1}, \ldots, A_{m}$ and let $\alpha_{1}, \ldots, \alpha_{m}$ denote the corresponding homomorphisms (4.9).

Let $A$ be a flat connection, and for simplicity assume that $A$ is irreducible. To work out the leading perturbative approximation to the contribution of the critical point $A$ to the path integral $Z_{M}(k)$, gauge fixing is needed. This is achieved by picking a Riemannian metric $g$ on $M$. Let $\alpha: \pi_{1}(M) \rightarrow G$ be the holonomy representation of $A$ and $\mathrm{Ad}: G \rightarrow \mathrm{GL}(\mathfrak{g})$ the adjoint representation. Then Ad $\circ \alpha$ is a representation

$$
\rho_{\alpha}: \pi_{1}(M) \longrightarrow \mathrm{GL}(\mathfrak{g})
$$

and we denote the associated flat bundle by $\mathfrak{g}_{\alpha}$. Since $\mathfrak{g}$ is compact, the Killing form is negative definite on $\mathfrak{g}$. Therefore, the negative of the Killing form defines a $G$-invariant inner product on $\mathfrak{g}$, and with respect to this choice of an inner product the representation (4.10) is orthogonal. Finally, we note that the De Rham complex $\Lambda^{*}\left(M ; \mathfrak{g}_{\alpha}\right)$ is equivalent to the complex of $\mathfrak{g}$-valued differential forms

$$
0 \rightarrow \Lambda^{0}(M ; \mathfrak{g}) \stackrel{d_{A}}{\longrightarrow} \Lambda^{1}(M ; \mathfrak{g}) \stackrel{d_{A}}{\longrightarrow} \Lambda^{2}(M ; \mathfrak{g}) \stackrel{d_{A}}{\longrightarrow} \Lambda^{3}(M ; \mathfrak{g}) \rightarrow 0
$$

where $\Lambda^{q}(M ; \mathfrak{g})=\Lambda^{q}(M) \otimes \mathfrak{g}$ and $d_{A}$ is the covariant derivative with respect to the connection $A$. Since $A$ is flat, we have $d_{A}^{2}=0$. By assumption, $A$ is irreducible and isolated modulo gauge equivalence. Therefore, we have $H^{0}\left(M ; \mathfrak{g}_{\alpha}\right)=H^{1}\left(M ; \mathfrak{g}_{\alpha}\right)=0$ and Poincaré duality implies $H^{*}\left(M ; \mathfrak{g}_{\alpha}\right)=0$, so that $\rho_{\alpha}$ is acyclic.

To describe the final result we need some more notation. Let $c_{2}(G)$ be the value of the Casimir operator of $G$ in the adjoint representation, normalized so that $c_{2}(\mathrm{SU}(N))=2 N$. Furthermore, let $I(g)$ be the Chern-Simons invariant of the Levi-Civita connection of $g$ with respect to a given trivialization of the tangent bundle of $M$, and let $\eta(g)$ be the $\eta$-invariant of the metric $g$.

Assume now that the representatives $A_{1}, \ldots, A_{m}$ of the gauge equivalence classes of flat connections are all irreducible. Then Witten's formula for the stationary phase approximation (or one loop approximation) of the path integral $(4.8)$ is

$$
Z_{M}(k) \sim \frac{1}{\# Z(G)} e^{i \pi d\left(\frac{1}{2} \eta(g)+\frac{1}{24 \pi} I(g)\right)} \sum_{j=1}^{m} e^{i\left(k+c_{2}(G) / 2\right) I\left(A_{j}\right)} \sqrt{T_{M}\left(\rho_{\alpha_{j}}\right)}
$$


where $Z(G)$ is the center of $G, d$ is the dimension of $G$, and $T_{M}\left(\rho_{\alpha_{j}}\right)$ is the analytic torsion of the flat connection $\rho_{\alpha_{j}}$. In fact, formula (2.23) in [W1] has to be slightly corrected (cf. [FG, (1.32)]).

Freed and Gompf [FG] have done explicit computations in a number of cases supporting the believe that (4.11) gives the correct asymptotic behaviour of Witten's invariant.

Since each $\rho_{\alpha_{j}}$ is acyclic, $T_{M}\left(\rho_{\alpha_{j}}\right)$ is independent of the choice of the metric $g$ on $M$ and, by [C, Mü], it coincides with the R-torsion $\tau_{M}\left(\rho_{\alpha_{j}}\right)$. Furthermore, by the Atiyah-Patodi-Singer theorem, $\frac{1}{2} \eta(g)+\frac{1}{24 \pi} I(g)$ is also independent of the metric. It depends only on the trivialization of the tangent bundle. As we know, the R-torsion $\tau_{M}\left(\rho_{\alpha_{j}}\right)$ can be computed from a triangulation $K$ of $M$ in a pure combinatorial way. This suggests that one may be able to develop a rigorous treatment of the path integral (4.8) on the combinatorial level and derive the asymptotic behaviour (4.11) in this way.

There exist also conjectures how (4.11) has to be modified if we give up the assumption that the gauge equivalence classes of flat connections are isolated and irreducible (cf., [FG, (1.36)]).

So far we considered the case of a compact gauge group. Witten has also started to investigate Chern-Simons theory with noncompact gauge group [W3]. This is of great interest, for example, in the application to 3-dimensional gravity. More precisely, $(2+1)$-dimensional gravity is related to Chern-Simons gauge theory with gauge group $\operatorname{SL}(2, \mathbf{C}), \operatorname{ISO}(2,1)$, or $\operatorname{SL}(2, \mathbf{R}) \times \operatorname{SL}(2, \mathbf{R})$ depending on whether the cosmological constant is positive, zero, or negative [W2]. For a general noncompact Lie group $G$, the quantization of Chern-Simons gauge theory w ith gauge group $G$ is not yet understood. For $\operatorname{SL}(2, \mathbf{R})$, however, the quantization is well understood $[\mathrm{K}]$. One can also study the perturbative expansion of the corresponding path integral [B-NW].

The perturbative treatment of Chern-Simons gauge theory with noncompact gauge group requires again gauge fixing. Since the Killing form is indefinite, there exists no obvious gauge fixing as in the compact case and different approaches are possible [B-NW]. For a semisimple Lie group $G$, the most natural gauge fixing seems to be the unitary gauge fixing described in [B-NW, §4]. Let $A$ be a flat connection on the trivial $G$-bundle over $M$ with holonomy representation $\alpha: \pi_{1}(M) \rightarrow G$. As above, let $\mathfrak{g}_{\alpha}$ be the flat bundle defined by $\rho_{\alpha}=\operatorname{Ad} \circ \alpha$. Then the unitary gauge fixing amounts to the choice of a Riemannian metric $g$ on $M$ and a Hermitian metric $h$ on $\mathfrak{g}_{\alpha}$. We observe that $\rho_{\alpha}: \pi_{1}(M) \rightarrow \mathrm{GL}(\mathfrak{g})$ is unimodular. In fact, since $\mathfrak{g}$ is semisimple, the Killing form is nondegenerate. Hence, for each $g \in G, \operatorname{Ad}(g)$ preserves a nondegenerate symmetric bilinear form on $\mathfrak{g}$ which implies that $|\operatorname{det} \operatorname{Ad}(g)|=1$. This is precisely the setting of $\S 2$.

Under the same assumption as above, one gets a formula for the one loop approximation of the path integral which is similar to (4.11). The analytic torsion $T_{M}\left(\rho_{\alpha_{j}}\right)$ is now defined by (2.2). For the discussion of the phase factor see $\S 4$ of [B-NW]. By assumption, each representation $\rho_{\alpha_{j}}$ is acyclic, and, therefore, by Corollary 2.7, $T_{M}\left(\rho_{\alpha_{j}}\right)$ is independent of the choice of the metric on $M$ and 
$\mathfrak{g}_{\alpha}$. Moreover, by Theorem 1, $T_{M}\left(\rho_{\alpha_{j}}\right)$ equals the R-torsion $\tau_{M}\left(\rho_{\alpha_{j}}\right)$ which has again a pure combinatorial describtion. This suggests that Chern-Simons gauge theory with a noncompact but semisimple gauge group should also be accessible to a combinatorial treatment.

\section{ACKNOWLEDGMENT}

This work was done during the author's visit to the Institute for Advanced Study in Princeton and the Max-Planck-Institut für Mathematik in Bonn, Germany. I am very grateful to both institutions for financial support and hospitality.

\section{REFERENCES}

[B-NW] D. Bar-Natan and E. Witten, Perturbative expansion of Chern-Simons theory with noncompact gauge group, preprint, IASSNS-HEP-91/4, Princeton, 1991.

[B-Z] J.-M. Bismut and W. Zhang, An extension of a theorem by Cheeger and Müller, Astérisque 205 (1992).

[BW] A. Borel and N. Wallach, Continuous cohomology, discrete subgroups, and representations of reductive groups, Ann. of Math. Stud., vol. 94, Princeton Univ. Press, Princeton, NJ, 1980.

[C] J. Cheeger, Analytic torsion and the heat equation, Ann. of Math. (2) 109 (1979), 259-322.

[FG] D. S. Freed and R. E. Gompf, Computer calculations of Witten's 3-manifold invariant, Comm. Math. Phys. 141 (1991), 79-117.

[K] T. P. Killingback, Quantization of $\mathrm{SL}(2, \mathbf{R})$ Chern-Simons theory, Comm. Math. Phys. 145 (1992), 1-16.

[MM] Y. Matsushima and S. Murakami, On vector bundle valued harmonic forms and automorphic forms on symmetric spaces, Ann. of Math. (2) 78 (1963), 365-416.

[Mi1] J. Milnor, Whitehead torsion, Bull. Amer. Math. Soc. 72 (1966), 358-426.

[Mi2] _ A duality theorem for Reidemeister torsion, Ann. of Math. (2) 76 (1962), 137-147.

[MS] H. Moscovici and R. J. Stanton, $R$-torsion and zeta functions for locally symmetric manifolds, Invent. Math. 105 (1991), 185-216.

[Mü] W. Müller, Analytic torsion and R-torsion of Riemannian manifolds, Adv. in Math. 28 (1978), 233-305.

[Mu] J. Munkres, Elementary differential topology, Ann. of Math. Stud., vol. 54, Princeton Univ. Press, Princeton, NJ, 1961.

[R] M. S. Raghunathan, On the first cohomology of discrete subgroups of semi-simple Lie groups, Amer. J. Math. 87 (1965), 103-139.

[RS] D. B. Ray and I. M. Singer, R-torsion and the Laplacian on Riemannian manifolds, Adv. in Math. 7 (1971), 145-210.

[RT] N. Yu. Reshetikhin and V. G. Turaev, Invariants of 3-manifolds via link polynomials and quantum groups, Invent. Math. 103 (1991), 547-597.

[S] A. Schwarz, The partition function of degenerate quadratic functional and Ray-Singer invariants, Lett. Math. Phys. 2 (1978), 247-252.

[Se] R. T. Seeley, Complex powers of an elliptic operator, Proc. Sympos. Pure Math., vol. 10, Amer. Math. Soc., Providence, RI, 1967, pp. 288-315.

[Wa] G. Warner, Harmonic analysis on semi-simple Lie groups. I, Springer-Verlag, Berlin, Heidelberg, and New York, 1972.

[W1] E. Witten, Quantum field theory and the Jones polynomial, Comm. Math. Phys. 121 (1988), 351-399. 
[W2] E. Witten, $2+1$ dimensional gravity as an exactly soluble system, Nuclear Phys. B 311 (1988/89), 46-78.

[W3] E. Witten, Quantization of Chern-Simons gauge theory with complex gauge group, Comm. Math. Phys. 137 (1991), 29-66.

Max-Planck-Institut für Mathematik, Gottfried-Claren-Str. 26, D-5300 Bonn 3, GerMANY

E-mail address: mueller@mpim-bonn.mpg.de 